\title{
Elucidating the cancer-specific genetic alteration spectrum of glioblastoma derived cell lines from whole exome and RNA sequencing
}

\author{
Vikas Patil'1,*, Jagriti Pal, ${ }^{1, *}$ and Kumaravel Somasundaram ${ }^{1}$ \\ ${ }^{1}$ Department of Microbiology and Cell Biology, Indian Institute of Science, Bangalore, India \\ * These authors have contributed equally to this work \\ Correspondence to: Kumaravel Somasundaram, email: ksomasundaram $1 @ g m a i l . c o m$ \\ Keywords: glioblastoma, exome \& RNA sequencing, cancer-specific mutations, gene fusions, RNA editing \\ Received: May 03, $2015 \quad$ Accepted: October 05, $2015 \quad$ Published: October 19, 2015
}

This is an open-access article distributed under the terms of the Creative Commons Attribution License, which permits unrestricted use, distribution, and reproduction in any medium, provided the original author and source are credited.

\section{ABSTRACT}

Cell lines derived from tumor tissues have been used as a valuable system to study gene regulation and cancer development. Comprehensive characterization of the genetic background of cell lines could provide clues on novel genes responsible for carcinogenesis and help in choosing cell lines for particular studies. Here, we have carried out whole exome and RNA sequencing of commonly used glioblastoma (GBM) cell lines (U87, T98G, LN229, U343, U373 and LN18) to unearth single nucleotide variations (SNVs), indels, differential gene expression, gene fusions and RNA editing events. We obtained an average of $41,071 \mathrm{SNVs}$ out of which 1,594 (3.88\%) were potentially cancer-specific. The cell lines showed frequent SNVs and indels in some of the genes that are known to be altered in GBM- EGFR, TP53, PTEN, SPTA1 and NF1. Chromatin modifying genes- ATRX, MLL3, MLL4, SETD2 and SRCAP also showed alterations. While no cell line carried IDH1 mutations, five cell lines showed hTERT promoter activating mutations with a concomitant increase in hTERT transcript levels. Five significant gene fusions were found of which NUP93-CYB5B was validated. An average of 18,949 RNA editing events was also obtained. Thus we have generated a comprehensive catalogue of genetic alterations for six GBM cell lines.

\section{INTRODUCTION}

Grade IV glioma or glioblastoma multiforme (GBM) is the most common and aggressive primary brain tumor. It accounts for $20 \%$ of all intracranial tumors [1] and comprises of neoplastic glial cells called astrocytes. GBM can arise via the de novo pathway without clinical or histologic evidence of a less malignant precursor lesion (primary GBM) or via the progressive pathway through development from a low-grade astrocytoma (secondary GBM) [2]. With the current mode of treatment of surgery along with temozolomide chemotherapy and radiotherapy, the median survival achieved till today is only 14.6 months [3].

Malignant tumors arise when genomic lesions accumulate within cells that disrupt normal cellular pathways ultimately giving them a survival advantage leading to tumor initiation, growth and metastasis. Each tumor carries a combination of genetic alterations that determine cancer prognosis and response to therapy. GBM tumors show significant amount of proliferation, invasion, angiogenesis and necrosis and is treatment refractory. In the past two decades, focused studies on candidate genes show various genetic alterations typical to GBM, e.g., TP53 mutation and loss, EGFR amplification and mutation, INK4a/ARF mutation, MDM 2/4 amplification or overexpression, PTEN mutation and loss of heterozygosity (LOH) in chromosome 10p and $10 q[4,5]$. In recent times, the advent of next generation sequencing (NGS) technologies has paved the path to analysis of entire cancer genome $[6,7]$. Whole exome sequencing (WES) and RNA sequencing (RNA-seq) are two techniques that can provide information for the functionally relevant part of the genome at increased coverage and reduced cost. Recently, two independent groups have carried out exome and RNA-seq analysis of GBM tissue samples and have found out various novel genetic alterations which may play important role in GBM 
Table 1: Whole exome and whole RNA sequencing statistics and quality assessment of glioma cell lines

\begin{tabular}{|c|c|c|c|c|c|c|c|}
\hline Cell line & U87 & T98G & LN229 & U343 & U373 & LN18 & Average \\
\hline \multicolumn{8}{|c|}{ I - Whole Exome Sequencing } \\
\hline $\begin{array}{l}\text { QC-passed } \\
\text { reads** }\end{array}$ & $41,937,382$ & $56,365,156$ & $59,066,920$ & $60,402,106$ & $48,108,386$ & $49,898,190$ & $52,629,690$ \\
\hline $\begin{array}{l}\text { Reads mapped } \\
(\%)\end{array}$ & 98.53 & 98.22 & 98.43 & 98.62 & 98.89 & 98.18 & 98.48 \\
\hline $\begin{array}{l}\text { Properly paired } \\
\text { reads }(\%)\end{array}$ & 97.97 & 97.41 & 97.44 & 97.69 & 98.33 & 96.52 & 97.56 \\
\hline $\begin{array}{l}\text { Target regions } \\
\text { covered }(\%)\end{array}$ & 99.50 & 99.80 & 99.70 & 99.50 & 99.80 & 99.80 & 99.68 \\
\hline $\begin{array}{l}\text { Average } \\
\text { coverage }(X) \text { for } \\
\text { whole exome } \\
\end{array}$ & 29.66 & 39.61 & 41.53 & 37.13 & 35.78 & 34.13 & 36.13 \\
\hline \multicolumn{8}{|c|}{ II - Whole RNA Sequencing } \\
\hline QC-passed reads & $33,796,760$ & $49,895,568$ & $42,609,412$ & $92,046,396$ & $47,085,884$ & $97,773,184$ & $60,534,534$ \\
\hline $\begin{array}{l}\text { Reads mapped ( } \\
\% \text { ) }\end{array}$ & 89.63 & 94.36 & 96.53 & 96.87 & 96.73 & 94.17 & 94.72 \\
\hline
\end{tabular}

*QC-passed reads $=$ reads having Phred quality score $\geq 30$

development and progression $[8,9]$.

Established cell lines from tumors play an important role as in vitro model to study various aspects of tumor development and progression. A comprehensive understanding of the genomic make-up of the cell lines will provide us with information regarding the alteration status of the genes present in the cell lines thus giving us an opportunity to choose the cell lines appropriately for particular studies. There have been three studies which characterized glioma derived cell lines using next generation sequencing [10-12]. However, these studies have carried out either whole genome or whole exome or RNA sequencing. Here, we have carried out an elaborate study to comprehensively characterize six GBM cell lines that are most commonly used. Both whole exome sequencing and whole RNA sequencing was carried out and in-depth analysis was performed to find out single nucleotide variations (SNVs), insertions/deletions (indels), transcriptional changes, gene fusions and RNA editing events. To our knowledge, this study is the first time an indepth characterization of the genomic alterations present in these cell lines have been carried out and we believe that this information will be highly useful to the scientific community.

\section{RESULTS}

\section{WES and RNA-seq statistics and quality assessment}

Genomic DNA from six GBM cell lines (U87, T98G, U343, LN229, U373, and LN18) was subjected to TruSeq exome capture and sequenced in Illumina
HiScanSQ platform (100 bp paired-end sequencing). Data analysis was carried out as given in Materials and Methods section. The raw reads were aligned to human reference genome hg19 and the initial quality statistics were assessed (Table 1). For each cell line, on an average $52,629,690$ reads passed quality criteria of Qscore (Phred quality score) $\geq 30$. While the average percentage of reads that mapped to hg19 was $98.48 \%$ across all cell lines, the average percentage of properly paired reads was $97.56 \%$. The targeted region (genomic regions covered by Illumina's exome capture kit) covered by the quality passed reads was $99.68 \%$. We obtained an average coverage of $\sim 36.31 \mathrm{X}$ which is suitable for calling variants with confidence as per Illumina guidelines [13].

Similarly, total RNA from the above six cell lines was subjected to RNA-seq in Illumina HiScanSQ platform (50 bp paired-end sequencing). The average number of reads obtained from each cell line came to be around $60,534,534$. The percentage of reads obtained from the sequencing that mapped to the human reference genome hg19 was $94.72 \%$ (Table 1). Given the fact that a minimum number of 25 million reads per sample is sufficient for RNA-seq data analyses, we found our samples with more than 33 million reads suitable for our study [14].

\section{Identification of single nucleotide variations (SNVs) and indels}

The variant calls were generated using GATK tool following the filtering criteria: 1) all SNVs detected should be restricted to the $62 \mathrm{Mb}$ region targeted by Illumina Truseq exome capture kit, 2) bases having quality score above 30 should be considered and 3) minimum 6 reads carrying variant bases should be present to be considered 
Table 2: Single nucleotide variation and indel classification and quantification

\begin{tabular}{|c|c|c|c|c|c|c|c|}
\hline Cell line & U87 & T98G & LN229 & U343 & U373 & LN18 & Average \\
\hline \multicolumn{8}{|c|}{ I. Single Nucleotide Variations (SNVs) } \\
\hline \begin{tabular}{ll|} 
Total \\
detected
\end{tabular} & 38,515 & 42,423 & 42,788 & 43,582 & 40,095 & 39,020 & 41,071 \\
\hline $\begin{array}{l}\text { Variants present in } \\
\text { dbSNP* }\end{array}$ & 37,545 & 41,029 & 41,196 & 42,001 & 38,915 & 37,224 & 39,652 \\
\hline Novel variants**: & 970 & 1,394 & 1,592 & 1,581 & 1,180 & 1,796 & 1,156 \\
\hline $\begin{array}{l}\text { Homozygous } \\
\text { variants }\end{array}$ & 19,710 & 20,762 & 21,293 & 17,104 & 22,175 & 23,566 & 20,768 \\
\hline $\begin{array}{l}\text { Heterozygous } \\
\text { variants }\end{array}$ & 18,805 & 21,661 & 21,495 & 26,478 & 17,920 & 15,454 & 20,302 \\
\hline Ti/Tv Ratio & 2.49 & 2.42 & 2.38 & 2.44 & 2.41 & 2.38 & 2.42 \\
\hline \multicolumn{8}{|l|}{ II. Indels } \\
\hline Total indels detected & 3,780 & 3,968 & 4,000 & 4,151 & 3,779 & 3,672 & 3,892 \\
\hline $\begin{array}{l}\text { Indels present in } \\
\text { dbSNP* }\end{array}$ & 3,528 & 3,693 & 3,712 & 3,831 & 3,515 & 3,390 & 3,612 \\
\hline Novel indels* & 255 & 275 & 288 & 320 & 264 & 282 & 280 \\
\hline Homozygous indels & 1,963 & 2,045 & 2,148 & 1,730 & 2,144 & 2,180 & 2,035 \\
\hline Heterozygous indels & 1,817 & 1,923 & 1,852 & 2,421 & 1,635 & 1,492 & 1,857 \\
\hline
\end{tabular}

*dbSNP refers to the Single Nucleotide Polymorphism database.

**In comparison with dbSNP Build 137.

as an SNV. We obtained an average number of 41,071 SNVs, out of which 39,652 SNVs were present in the single nucleotide polymorphism database (dbSNP) 137 build while 1,156 were novel SNVs (Table 2). Further, we found an average of 20,768 homozygous and 20,302 heterozygous SNVs. The average transition vs transversion ( Ti/Tv) ratio was 2.42 (Table 2) [10].

Small insertions-deletions (indels) were identified with GATK tool using similar filtration criteria as used for SNVs. The list of indels found from WES is given in Supplementary Table 1. Indels were observed to range in size between -49 to +29 bases (Supplementary Figure $\mathrm{S} 1 \mathrm{~A})$ and were detected at a proportion of 10 to $12 \%$ of that of SNVs (Table 2) [15]. The average number of indels detected was 3,892 out of which 3,612 were present in dbSNP build 137 while 280 were novel (Table 2 ). Although the indels distribution followed power law distribution, there was small deviation at 4-base indels (Supplementary Figure S1A) [16]. Interestingly, when the indel distribution in the coding region alone was looked into, there was an enrichment of indels of size equal to multiples of three bases (Supplementary Figure S1B).

Next, we classified the SNVs and indels using Oncotator [17], according to their location in the genome and also the type of changes the alteration will bring to the protein (Supplementary Figure S1C, D, E, F). While $\mathrm{SNV}$ s were found in equal proportion between non-coding and protein coding regions, a tenfold more occurrence of indels in non-coding region compared to protein coding regions was found (Supplementary Figure S1C and D). We found a high occurrence of SNVs in the form of missense and silent mutations compared to a very low occurrence in the form of nonsense, nonstop, splice site and translation start site mutation (Supplementary Figure S1E). Indels resulted in frame-shift, in-frame and splice site changes at a higher frequency, but it was found rarely in translational start sites. (Supplementary Figure S1F).

\section{Comparison of SNVs from exome data to catalogue of somatic mutations in cancer (COSMIC) and cancer cell line encyclopedia (CCLE) databases}

COSMIC database contains most comprehensive resource of genetic alterations occurring in a large number of human cancer tissue and cancer derived cell lines [18]. CCLE project undertaken by Broad Institute provides information for mutations, copy number variations (CNVs) and mRNA expression in a large panel of cell lines [19]. To test the robustness of in-house WES data, we compared SNVs detected in this study with COSMIC and CCLE databases. SNV information was available for four of the six cell lines we have studied (U87, T98G, LN229 and LN18) in COSMIC and CCLE databases. We found that our data had an average concordance of $65.3 \%$ with COSMIC and 74.74\% with CCLE databases (Supplementary Figure S2). A more in-depth comparison of cancer specific mutations between our finding and COSMIC database is described later (Figures 3 and 4). 


\section{Detection of cancer-specific SNVs from WES data}

Due to unavailability of matched normal samples and also to eliminate the mutations which may have no role in cancer development and progression, a stringent filtration criteria was followed as described in Materials and Methods section and the SNVs were divided into cancer-specific and non-specific types (Supplementary Figure S9). The list of cancer-specific SNVs is given in Supplementary Table 2. The average percentage of cancer-specific SNVs for each cell line was found to be $3.88 \%$ of the total number of SNVs obtained. The average number of cancer-specific and non-specific SNVs across the cell lines came to be approximately 1,595 and 39,476 respectively (Figure 1A). The number of non-specific SNVs was quite variable for each cell line, although the number of cancer-specific SNVs showed more or less equal frequencies. The cell line LN18 showed a higher cancer-specific SNV rate compared to its relatively lower non-specific SNVs. Previous reports identified that cell lines with microsatellite instability (MSI), which arises due to mutation in mismatch repair (MMR) genes, harbored several fold higher cancer-specific mutations compared to those cell lines without MSI [11]. In our study, the frequency of cancer-specific mutations in all the six cell lines was in a much lower range $(1595 \pm$ 310 ), same as that of cell lines without MSI as previously reported [11]. Concomitantly, we also found that none of cell lines studied here harbored mutation in MMR genes (MSH2, MSH3, MSH6, MLH1, PMS2, MSH4, MSH5, MLH3, PMS1 and PMS2L3). This is expected because microsatellite instability is a rare phenomenon in GBM [20].

The percentages of homozygous and heterozygous SNVs for cancer-specific changes have been represented (Figure 1B). The ratio of the homozygous to the heterozygous variants was around 1:8. Somatic mutations

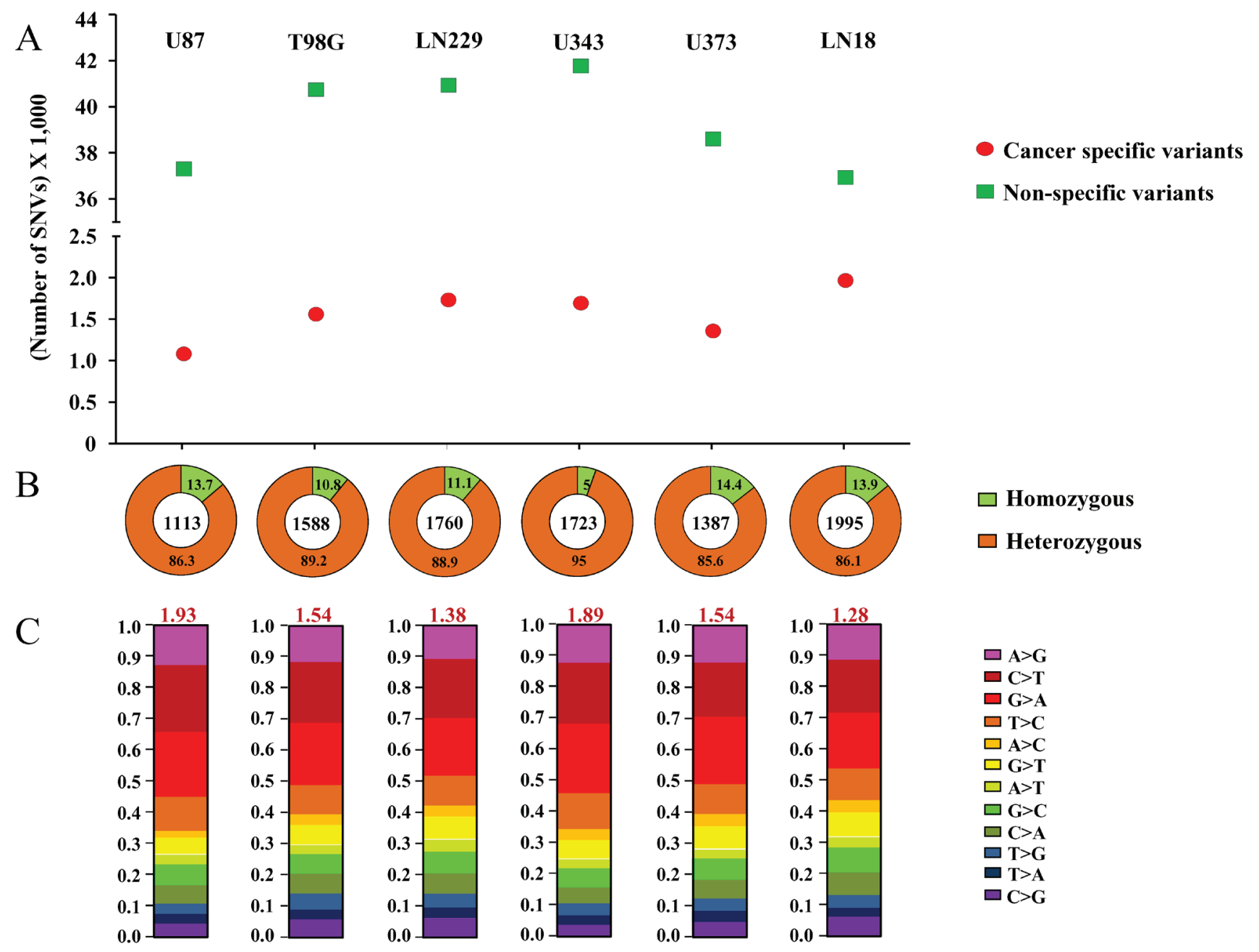

Figure 1: Cancer-specific mutation quality statistics: A. Number of Cancer-specific versus non-specific SNVs. B.. Percentage of homozygous versus heterozygous changes. The numbers in each quadrant represent the percentage, and the number in the center of the circle represents the total number of cancer-specific mutations. C. Ti/Tv changes for each cell line's cancer-specific SNVs. Each type of base change has been given by different color codes and the overall Ti/TV ratio has been given in red font at the top of each bar-plot. For A., B. and C., plot for each cell line has been given in one column and the name of the cell line has been specified at the top. 
being sporadic and random in nature, the probability of both alleles being mutated similarly is rare which is why cancer-specific SNVs show a very high proportion of heterozygous changes as compared to homozygous changes. The proportion of each type of base changes for the cancer-specific SNVs for each cell line has been given (Figure 1C). The average Ti/Tv ratio for each cell line came to be around 1.66 (ranging from 1.28 to 1.93 ), in a similar range as reported before [11]. The proportion of each functional type of SNVs, depending upon their genomic location or the consequence that the SNV can bring about in the gene product i.e. changes in exonic regions, has been plotted for both types of variants (Figure 2). It was observed that the proportion of cancer-specific SNVs is higher in regions where nucleotide changes have a deleterious consequence. This includes the functional classes 'missense mutation' and 'nonsense mutation', as previously reported, as compared to other regions where non-specific SNV proportion was observed to be more [11].

\section{Comparison of indels and cancer-specific SNVs with TCGA, COSMIC and CCLE databases}

We investigated the overlap between mutations (indels and cancer-specific SNVs) identified in the six cell lines and the significantly mutated genes in GBM tumor samples as per large scale exome sequencing $[8,9]$. Figure $3 \mathrm{~A}$ shows the map of occurrence of alterations in these genes for the six cell lines studied. Out of 38 genes tested, we found mutations in 12 genes in one or more cell lines from WES. As previously reported, the genes TP53,
PTEN and EGFR are mutated in more than $20 \%$ of TCGA GBM tumor tissue samples [8,9]. We found PTEN and TP53 to be mutated in 4 out of 6 cell lines, while EGFR was mutated in one cell line only (Figure 3A). Among the genes that are mutated between 5 to $20 \%$ in TCGA GBM tumor samples [8, 9], we found mutations in NF1, SPTA1, TCHH and ATRX genes, although, the other genes like PIK3R1, PIK3CA, RB1, IDH1 and KEL were not mutated in any cell line (Figure 3A). Upon analyses of the status of a set of 34 chromatin modifying genes [9], we found mutations in ATRX, SETD2, SRCAP, MLL3 and MLL4, genes (Figure 3B). Investigation of the mutation status of DNA repair genes that were mutated in TCGA study revealed that out of 61 genes that were analysed, 17 genes harbored SNVs in one or more cell lines (Figure 4). No indels were observed in the DNA repair genes. No mutation was observed in any of the genes related to base excision repair (BER) and also, in MMR genes (Figure 4).

We also investigated the overlap between indels and cancer-specific SNVs with that of COSMIC and CCLE databases for four cell lines which are common between these data sets [18] (Figures 3 and 4). A total of 32 mutations were uncovered in 129 genes tested from WES in U87, T98G, LN229 and LN18 (Figures 3 and 4) out of which 21 mutations were reported either in COSMIC or CCLE or both datasets giving a concordance of $65.6 \%$, and the rest 11 mutations were found novel from WES data (Supplementary Table S3). Further, 13 novel mutations were also observed in the remaining two cell lines, U343 and U373 (Figures 3 and 4; Supplementary Table 3). From the 24 novel mutations found across all six cell lines, 8 were selected for validation by Sanger sequencing out of which 7 could be validated (Supplementary Figure S3).

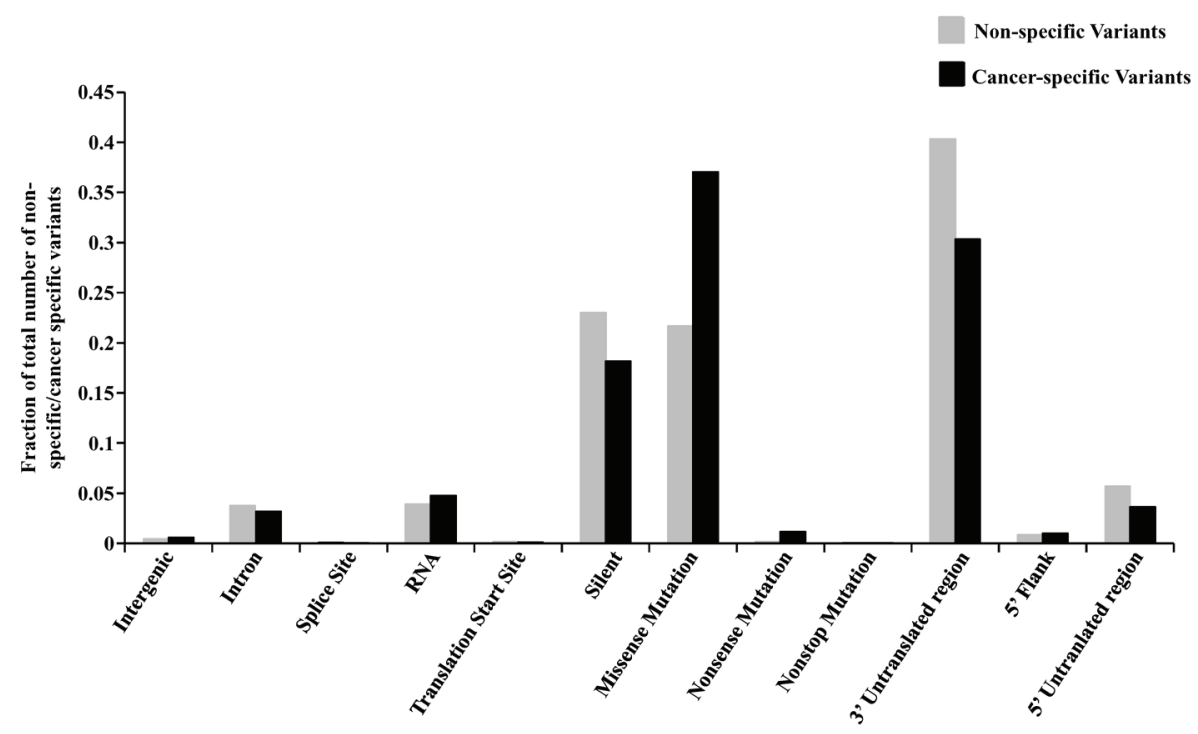

Figure 2: Functional classification of cancer-specific versus non-specific variants. The variants have been plotted as a fraction of the total number of cancer-specific (CS) or non-specific (NS) variants. Fraction of CS and NS variants have been plotted for each functional type according to their location in the genome i.e. inter-genic region, intron, 5' flank, 5' UTR, 3' UTR, RNA, splice site, translation initiation site and coding region which has been further subdivided into the type of change the alteration will bring to the protein (missense, nonsense, non-stop and silent). 


\section{Functional importance of cancer-specific SNVs}

The cancer-specific SNVs obtained in this study might have potential carcinogenic consequence as these changes are not observed in multiple normal human sequence databases. Out of 129 genes as in Figures 3 and 4, 30 genes were seen to harbor non-synonymous cancer-specific SNVs in one or more cell lines. We used PolyPhen-2 and SIFT tools on the above 30 genes to predict whether a particular cancer-specific SNV can alter the function of the protein significantly [21, 22]. Mutations that were predicted by both tools to be deleterious or damaging were considered. Thirty percent of genes $(n=9)$ were found to contain SNVs that attribute a significant change in protein function (Supplementary Table S4). The functional significance of the SNVs present in NF1, MLL3, BRCA2, ATR, ERCC2 and TP53 was further investigated. The protein domain structure and the positions mutated in each protein is given (Supplementary
Figure S4). NF1 is a negative regulator of RAS signaling pathway. It has a RAS-GAP domain (1174-1535 amino acid positions) which functions to convert GTP in activated RAS to GDP, thus inactivating RAS. Recurrent mutations are found in GBM tumor in NF1 RAS-GAP domain [9]. NF1 mutation in U87 occurs in this domain where lysine residue at 1444 position gets converted to methionine which could lead to abrogation of GTPase activity of NF1 [23]. The tumor suppressor gene MLL3 is mutated in three positions, G892R, Y987H and C988F, in different cell lines (Supplementary Table 4). Of the three positions, $\mathrm{Y} 987 \mathrm{H}$ and $\mathrm{C} 988 \mathrm{~F}$ are reported in COSMIC database [18]. MLL3 is a nuclear protein comprising of 6 PHD finger domains, one of each of HMG, FYRN, FYRC and SET domains [24] and it functions as a histone methyl transferase. In cancer scenario, it has been observed that enrichment of mutations is present in the PHD, FYNC, FYRC and SET domains [25]. The mutations, Y987H and C988F, found in the cell lines are present in PHD finger
A

SNVs novel in in-house data

SNVs present in Cosmic only

SNVs present in CCLE only

SNVs present in Cosmic and in-house data

SNVs present in Cosmic and CCLE

SNVs present in Cosmic CCLE and in-house data

Frameshift indel in Cosmic, CCLE and in-house data

Frameshift indel novel in inhouse data

Inframe indel novel in inhouse data

Inframe indel in Cosmic and CCLE

Frameshift indel in Cosmic only

Frameshift indel in CCLE only

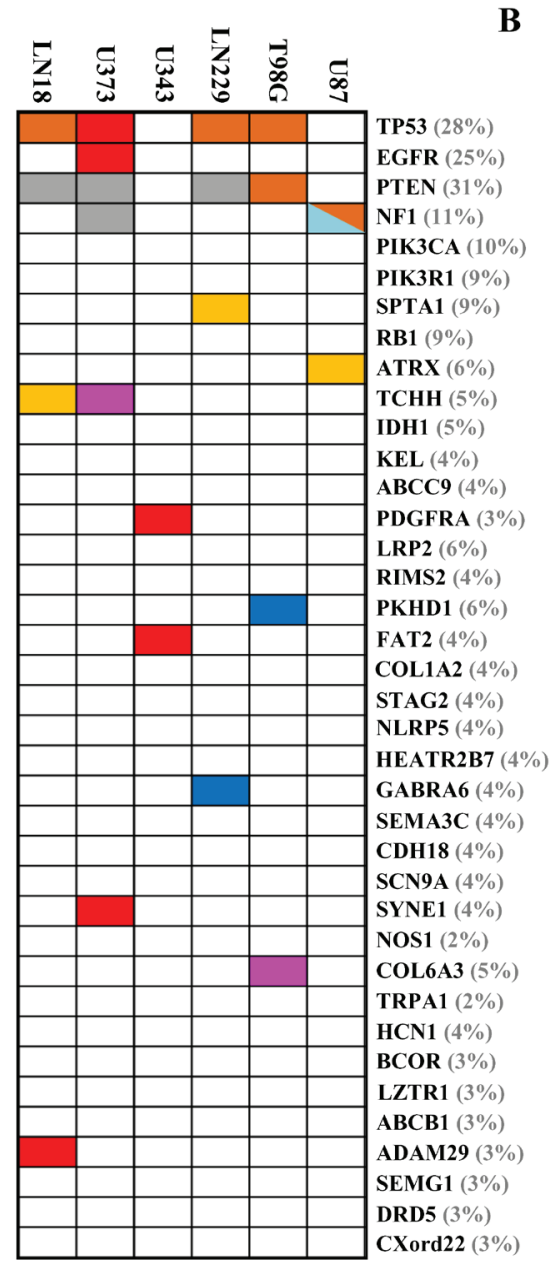

B

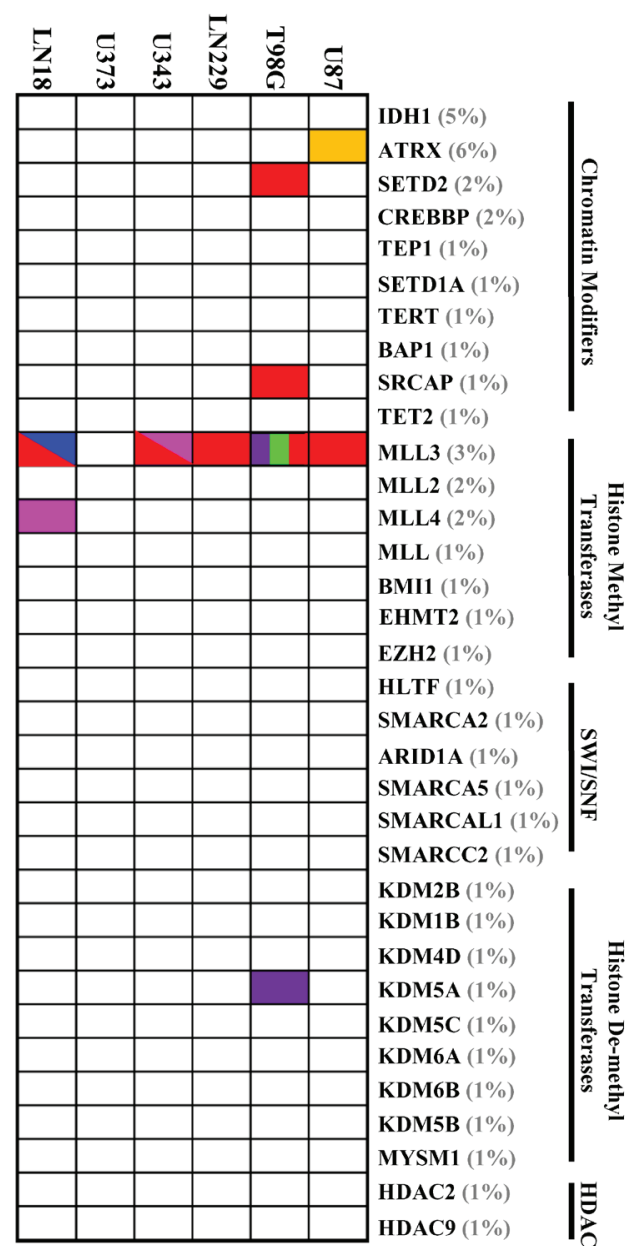

Figure 3: Mutation spectrum for most commonly mutated genes and chromatin modifying genes in each cell line. A. SNV and indel status of genes $(n=38)$ in cell lines that are frequently altered in GBM $[8,9]$. B. SNV and indel status of frequently altered chromatin modifying genes $(n=34)$ [9]. The percentage at which each gene is mutated in TCGA GBM tissue [9] has been provided in brackets after each gene name. Comparison with COSMIC \& CCLE databases for the same genes A. and B. is also shown. The dual/triple colors indicate that the gene harbors two/three types of mutations in the particular cell line respectively. 
domain of MLL3. PHD finger domains are required for proper histone methylation [25] and mutation in this domain will significantly abrogate MLL3 function. LN18 cell line was seen to harbor a deleterious mutation in BRCA2 (N856Y). BRCA2 interacts with various proteins like RAD51, PALB2, NPM1, PCID2 and DSS1 and is mainly involved in DNA double strand break repair. The above mutation in BRCA2 is present in the region which is involved in binding to NPM1 [26]. ATR is a kinase that gets activated when DNA damage occurs within the cell. LN229 cell line was seen to harbor G2375R mutation in the kinase domain (2321-2567 amino acid positions) of ATR which might severely abrogate its function [27]. ERCC2, mutated in LN18 (R690W), is a nuclear protein involved in the repair of damaged DNA through nucleotide excision repair pathway. This particular mutation is present in the nuclear localization signal sequence (682696 amino acid positions) of ERCC2 and this will hamper the targeting of ERCC2 to the nucleus [28]. The tumor suppressor gene, TP53, is involved in the regulation of apoptosis, genome stability and angiogenesis and it is found to be mutated in almost all types of cancer. In this study, 4 out of 6 cell lines was found to be mutant for TP53 (T98G, LN229, U373 and LN18) where the mutations were seen to be present in the DNA binding domain (95289 amino acids), thus leading to significant functional alteration in the protein. Since TP53 is a transcriptional regulator, its mutation will have a direct effect on the transcriptome of the cell.

We next sought to find out the alteration in the transcriptome in p53 mutant versus p53 wild-type cell lines from RNA-seq data. The expression levels of 227 genes were found to be altered in p53 mutant cell lines as compared to p53 wild type ones (Supplementary Table 5). These 227 genes were subjected to analysis using DAVID tool to find out pathways enriching in p53 mutant conditions (Supplementary Figure S5) [29, 30]. p53 regulates multiple processes within the cell which include cell differentiation [31-33], neuron development [34-36], cell adhesion [37-40], cell proliferation and apoptosis [41-47]. All these biological processes were seen to be significantly enriched when above 227 genes were subjected for pathway enrichment in DAVID Gene Ontology (GO) analysis (Supplementary Figure S5).

We next investigated the mutation status of isocitrate dehydrogenase genes (IDH1 and IDH2) in the cell lines. Mutation in IDH1 is observed in $\sim 80 \%$ of grade 2 and 3 gliomas and secondary GBM [48, 49]. The typical mutation that occurs in gliomas is $\mathrm{R} 132 \mathrm{H}$ mutation, although $\mathrm{R} 132 \mathrm{C}$ or $\mathrm{R} 132 \mathrm{~S}$ is also seen at much lower percentages [50]. Paralog of IDH1, i.e., IDH2 mutated in the position R172 also show similar effect although

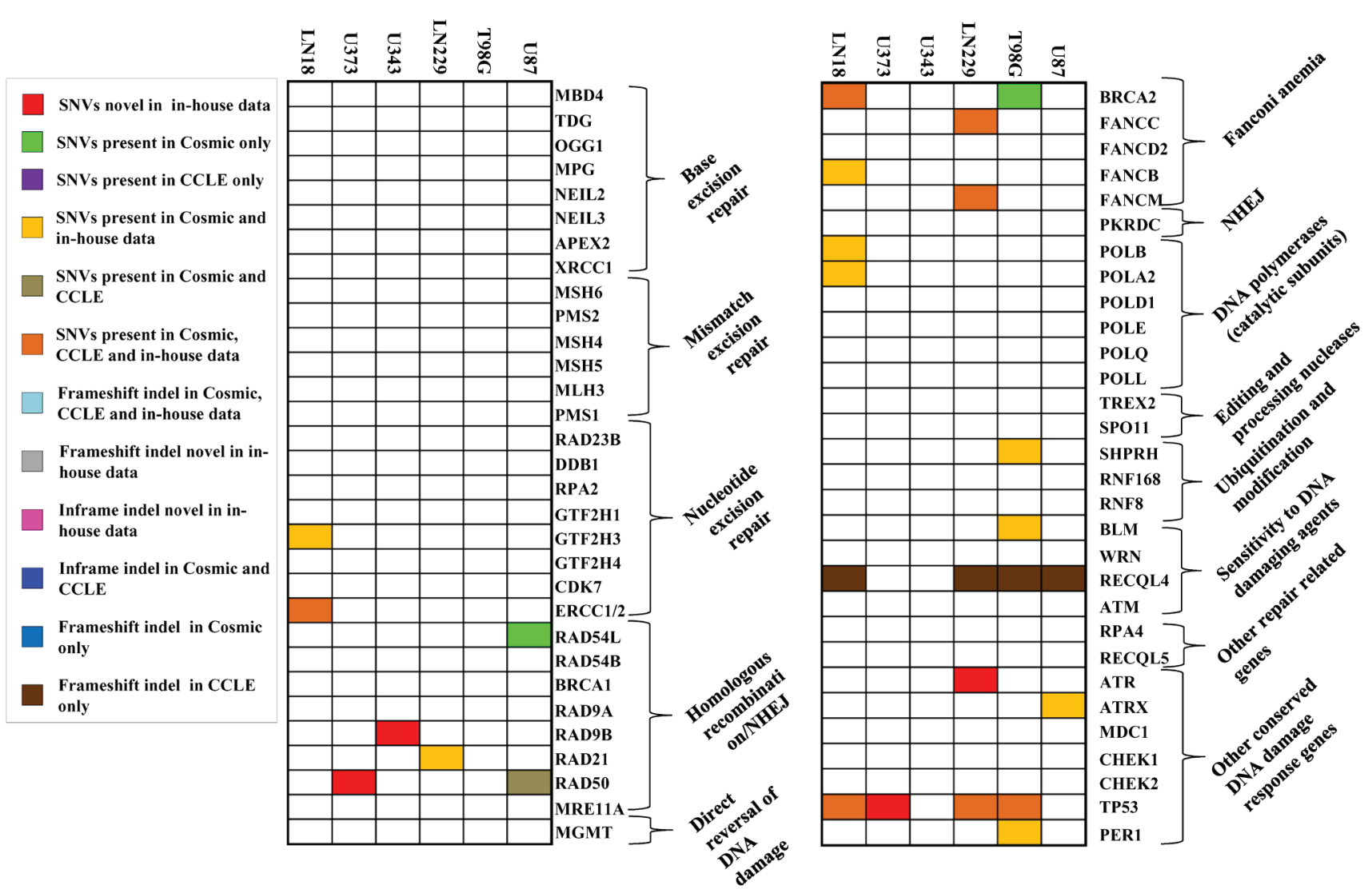

Figure 4: Mutation spectrum for DNA repair genes in each cell line. SNV and indel status of genes $(n=61)$ in cell lines that are altered in GBM [9]. Comparison with COSMIC \& CCLE databases for the same genes is also shown. 
frequency of mutation in IDH2 is rare [51]. No mutations in IDH1 or IDH2 were obtained from WES data in the cell lines tested. IDH1 mutation status was further validated through Sanger Sequencing, which included an additional three cell lines - U251, SVG and immortalized human astrocytes (IHA) (Figure 5A; Supplementary Figure S6A). Thus the cell lines used are derived from primary GBM patients.

Telomerase activation caused by promoter mutation in glioma has been reported recently [52-54]. There are two mutations, $\mathrm{C}>1,295,228>\mathrm{T}$ and $\mathrm{C}>1,295,250>\mathrm{T}$ reported in the promoter region of hTERT gene. Fifty five percent of GBM tumor samples harbor hTERT promoter mutation where the above two mutations are mutually exclusive [54]. Since the exome enrichment method used does not capture the hTERT promoter region, we carried out Sanger sequencing to detect hTERT promoter mutations. U87, LN229, U343, U251, and U373 cell lines showed C228T whereas; T98G showed C250T promoter activating mutations (Figure 5B; Supplementary Figure S6B). GBM cell line LN18 and the immortalized normal astrocytes SVG and IHA showed no mutation in hTERT promoter. RT-qPCR for hTERT mRNA revealed increased levels in cell lines carrying hTERT promoter mutations in GBM cell lines (U87, LN229, U343, U251, U373 and T98G) and IHA in comparison to wild type cell lines (LN18 and SVG) (Figure 5C). IHA, harboring wild-type hTERT promoter, shows high levels of the mRNA because the immortalization of the cell line has been carried out by overexpression of hTERT along with E6/E7 viral proteins. From the above investigations, it is evident that mutations present in functionally important part of the proteins indeed lead to drastic changes in their functions which ultimately play a role in carcinogenesis.
A

\begin{tabular}{|l|c|}
\hline Sample & R132H/C/S \\
\hline U87 & No \\
\hline T98G & No \\
\hline LN229 & No \\
\hline U343 & No \\
\hline U251 & No \\
\hline U373 & No \\
\hline LN18 & No \\
\hline SVG & No \\
\hline IHA & No \\
\hline
\end{tabular}

$\mathrm{B}$

\begin{tabular}{|l|c|c|}
\hline Cell Line & $\begin{array}{c}\mathbf{1 , 2 9 5 , 2 2 8} \\
(\mathbf{C 2 2 8})\end{array}$ & $\begin{array}{c}\mathbf{1 , 2 9 5 , 2 5 0} \\
(\mathbf{C 2 5 0 T})\end{array}$ \\
\hline U87 & Mutated & Not mutated \\
\hline T98G & Not mutated & Mutated \\
\hline LN229 & Mutated & Not mutated \\
\hline U343 & Mutated & Not mutated \\
\hline U251 & Mutated & Not mutated \\
\hline U373 & Mutated & Not mutated \\
\hline LN18 & Not mutated & Not mutated \\
\hline SVG & Not mutated & Not mutated \\
\hline IHA & Not mutated & Not mutated \\
\hline
\end{tabular}

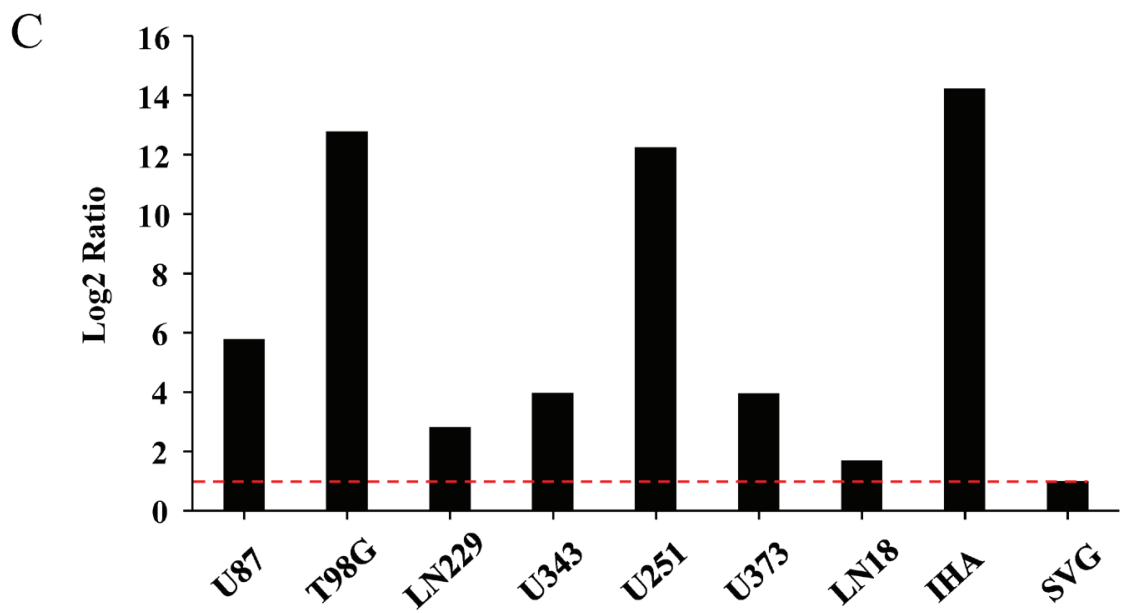

Figure 5: Mutation status of IDH1 and hTERT promoter in the cell lines. A. Mutation status of IDH1 position R132H/C/S in each cell line. B. Presence or absence of C228T or C250T mutations in the promoter region of hTERT in each cell line. C. Real-time PCR quantification of hTERT mRNA in different cell lines has been given. The mRNA level for SVG has been normalized to 1 and the levels for other cell lines have been given in comparison to that of SVG. Red line indicates 1 in $\log 2$ scale. 
Gene expression of GBM cell lines and comparison with GBM tumor transcriptome

Differential gene expression profiling was carried out by comparing RNA-seq data of the six cell lines with that of five normal brain tissue samples from TCGA. As per Gencode Version 19 annotation file [55], applying a cut-off of 2 fold change in absolute expression value, we obtained a total of 3,428 differentially regulated genes of which 509 were up regulated and 2,919 were down regulated (Figure 6A and 6B). The differentially expressed genes were classified into the different classes of RNA - protein coding, long non coding RNA and miRNA etc. (Figure 6A) and the entire result was tabulated (Supplementary Table 6).

The differentially regulated genes in the six GBM cell lines were compared with Agilent microarray data of GBM tumor tissue samples from TCGA (Figure 6C). Of the 3,428 genes differentially regulated in the cell lines

A

\begin{tabular}{|l|c|c|}
\hline \multicolumn{1}{|c|}{ Type } & Up & Down \\
\hline protein_coding & 427 & 2226 \\
\hline antisense & 29 & 169 \\
\hline lincRNA & 19 & 329 \\
\hline sense_overlapping & 1 & 21 \\
\hline sense_intronic & 1 & 11 \\
\hline processed_transcript & 4 & 19 \\
\hline pseudogene & 24 & 116 \\
\hline polymorphic_pseudogene & 0 & 3 \\
\hline miRNA & 2 & 10 \\
\hline misc_RNA & 0 & 3 \\
\hline rRNA & 1 & 1 \\
\hline snRNA & 0 & 3 \\
\hline snoRNA & 1 & 2 \\
\hline 3 prime_overlapping_ncrna & 0 & 3 \\
\hline TR_C_gene & 0 & 3 \\
\hline Total & 509 & 2919 \\
\hline
\end{tabular}

$\mathrm{C}$

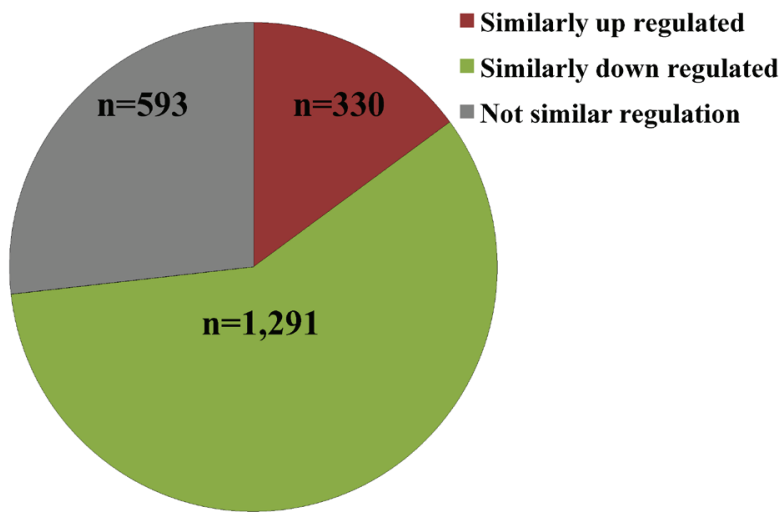

2,214 genes (383 up regulated and 1,831 down regulated) were found to be present in Agilent microarray gene list from TCGA GBM tumor samples. Differentially expressed genes from TCGA's Agilent microarray data was selected on the basis of $p$-value cut-off ( $p$-value $\leq 0.05$ ). A total of 1,621 genes were found to be similarly regulated between in-house cell line data and TCGA GBM Agilent data (Figure $6 \mathrm{C}$ ) thus giving a concordance of $\sim 73 \%$.

\section{Analysis of potential oncogenic gene fusions from RNA-seq data}

Numerous studies discovered gene fusion to be a critical and significant event in hematopoietic tumors [56] as well as solid cancers including GBM [8, 9, 5762]. In the six cell lines analyzed here, a total of 389 gene fusion events were detected using three different tools: FusionMap [63], TopHat-Fusion [64] and PRADA [65] (Supplementary Table 7), out of which 5 fusions were

B

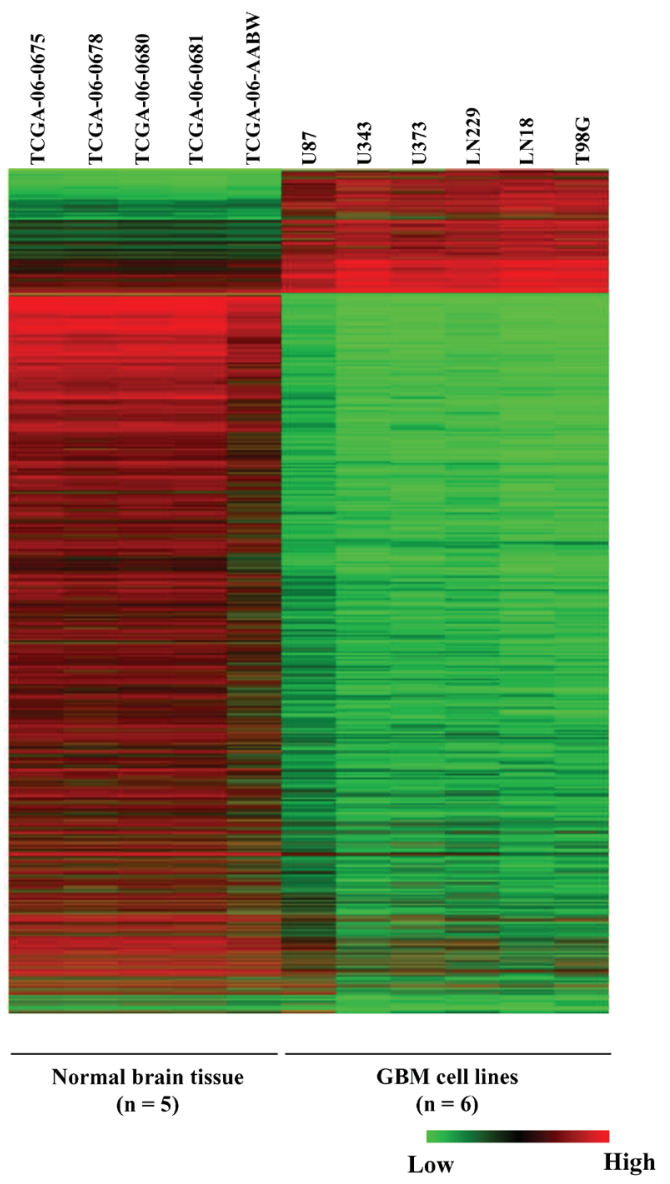

Figure 6: Gene expression analysis of GBM cell lines. A. Distribution of different classes of RNAs that are differentially regulated across the six GBM cell lines compared to normal brain samples. B.. Heat map of the differentially expressed genes in the six GBM cell lines versus normal brain samples. C. Concordance of the transcriptome data from RNA-seq data of cell lines with Agilent microarray of GBM samples from TCGA. Of the 3,428 differentially regulated genes from cell lines, concordance in 2,214 genes that are also present in Agilent microarray data has been presented. 
detected by all three tools (Figure 7A and 7B). A low overlap between the three tools was obtained because PRADA uses stringent criteria for fusion detection and hence only few fusions were obtained from PRADA. Only two of the five fusions namely NUP93-CYB5B and STX17-NR4A3 were found to give rise to in-frame fusion products and both were present in LN18 cell line (Figure 7B).

NUP93-CYB5B gene fusion was taken up for characterization. As per analysis by PRADA, NUP93CYB5B fusion was represented by a total of 19 junctionspanning reads (Figure $7 \mathrm{~B}$ ). Both the fusion partners are located in the same chromosome (Chromosome 16) where NUP93 has 20 exons and CYB5B has 5 exons (Figure 7B). The predicted reading frame at the breakpoint is shown for NUP93 (green) and CYB5B (purple) fusion (Figure 7C). The predicted intron-exon structure of the fused product shows that the first three exons of
NUP93 and last four exons of CYB5B are retained and the fusion is created by joining of the end of exon 3 of NUP93 with the start of exon 2 of CYB5B (Figure 7C and 7D). The genomic breakpoint was mapped to $\mathrm{Chr}$. $16 q 13$ position 56,839,544 for NUP93 and Chr. 16q22.1 position $69,481,053$ for CYB5B (Figure 7D). NUP93 is a nucleoporin protein comprising of 818 amino acids and it plays a vital role in nuclear pore complex formation (Figure 8A). While its C-terminal amino acids ( 600) are essential for the assembly of the structural backbone of the nuclear pore complexes [66], the N-terminal 165 amino acids are required for binding to CREB-binding protein (CBP) [67](Figure 8A). The C-terminal region of CYB5B, a mitochondrial hemoprotein, is essential for its localization to the outer mitochondrial membrane (Figure 8A) [68]. Analysis revealed that the fusion protein encoded by fused transcript is a 222 amino acid protein consisting of the N-terminal 163 amino acids of NUP93
A

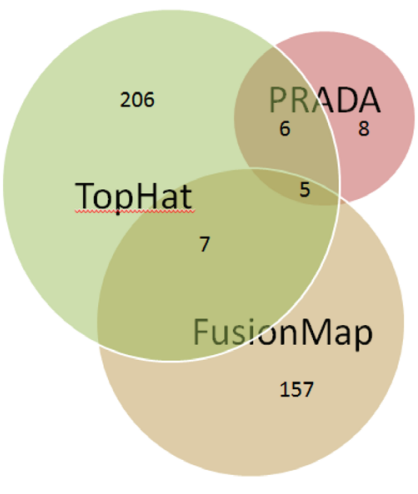

C

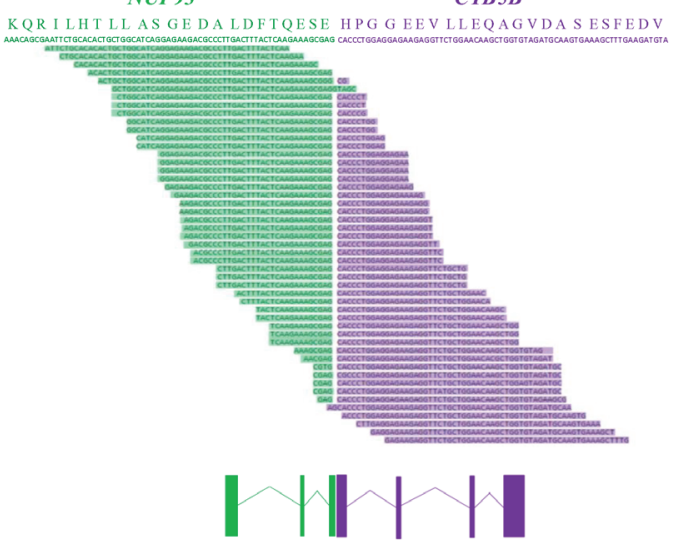

$\mathrm{B}$

\begin{tabular}{|c|c|c|c|c|c|c|c|c|}
\hline $\begin{array}{l}\text { Sr. } \\
\text { No }\end{array}$ & $\begin{array}{l}\text { Cell } \\
\text { Line }\end{array}$ & & Gene & Frame & \begin{tabular}{|c|} 
Junction \\
Spanning \\
Reads
\end{tabular} & Chr & $\begin{array}{c}\text { Numbers } \\
\text { of exons }\end{array}$ & $\begin{array}{c}\text { Position of fusion } \\
\text { in exon }\end{array}$ \\
\hline \multirow{2}{*}{1} & \multirow{2}{*}{ LN18 } & 5'gene & NUP93 & \multirow{2}{*}{ In-frame } & \multirow{2}{*}{19} & 16 & 20 & End of exon 3 \\
\hline & & 3'gene & CYB5B & & & 16 & 5 & Start of exon 2 \\
\hline & & & & & & & & \\
\hline \multirow{2}{*}{2} & \multirow{2}{*}{ LN18 } & 5'gene & STX17 & \multirow{2}{*}{ In-frame } & \multirow{2}{*}{5} & 9 & 8 & End of exon 3 \\
\hline & & 3'gene & NR4A3 & & & 9 & 7 & Start of exon 5 \\
\hline & & & & & & & & \\
\hline \multirow{2}{*}{3} & \multirow{2}{*}{ U343 } & 5'gene & TNS3 & \multirow{2}{*}{$\begin{array}{l}\text { 5UTR- } \\
\text { 5UTR }\end{array}$} & \multirow{2}{*}{14} & 7 & 31 & End of exon 3 \\
\hline & & 3'gene & TBRG4 & & & 7 & 11 & Start of exon 2 \\
\hline & & & & & & & & \\
\hline \multirow{2}{*}{4} & \multirow{2}{*}{ U343 } & 5'gene & C19orf25 & \multirow{2}{*}{$\begin{array}{l}\text { CDS- } \\
\text { 5UTR } \\
\end{array}$} & \multirow{2}{*}{13} & 19 & 3 & End of exon 2 \\
\hline & & 3'gene & CSNK1G2 & & & 19 & 12 & Start of exon 2 \\
\hline & & & & & & & & \\
\hline \multirow{2}{*}{5} & \multirow{2}{*}{ T98G } & 5'gene & PTDSS2 & \multirow{2}{*}{$\begin{array}{c}\text { not- } \\
\text { classified }\end{array}$} & \multirow{2}{*}{6} & 11 & 12 & End of exon 2 \\
\hline & & 3'gene & RAB20 & & & 13 & 2 & Start of exon 2 \\
\hline
\end{tabular}

$\mathrm{D}$

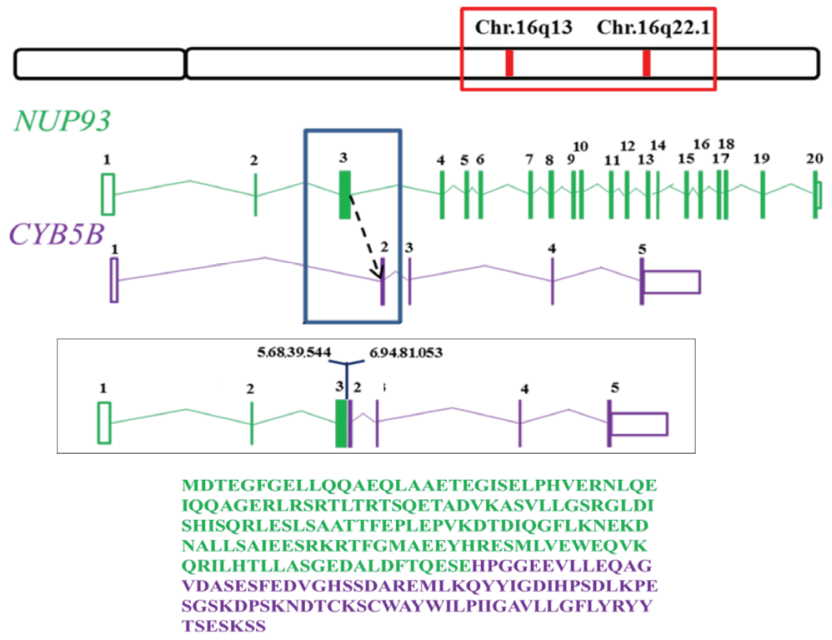

Figure 7: Gene fusion analysis. A. Number of fusions obtained from each tool- TopHat-Fusion, FusionMap and PRADA. Intersection of each circle represents the fusions found common in between the tools. B. Five fusions obtained from all three tools specifying cell line in which it is present, chromosome no., fusion type, total no. of exons for each gene. C. Split reads aligning to the gene fusion junction as per TopHat-Fusion prediction. The possible protein sequence present at the junction has been given on top with NUP93 sequence represented in green and CYB5B sequence in purple. The exonic structure of the gene fusion is given at the bottom where boxes represent exons and the lines represent introns. D. Chromosomal location of the members of the fusion, exon-intron structure and possible fusion region has been depicted. The entire fusion protein sequence has been provided where amino acids represented by green letters belong to NUP93 and those represented by purple belongs to CYB5B. 
and C-terminal 59 amino acids of CYB5B (Figure 7D and $8 \mathrm{~A}$ ). Thus the fusion protein is likely to target the CBP to the mitochondria with a potential possibility of acetylation of certain proteins located in the mitochondrial outer membrane. Indeed, acetylation of mitochondrial enzymes present in the mitochondrial matrix occurs in nutrient excess condition thus leading to their inactivation [69]. Mitochondrial lysine acetyltransferase has not been identified till date although it has been observed that knockdown of a homolog of histone acetyltransferase in yeast leads to hypoacetylation in mitochondrial enzymes [70]. Acetylation of outer mitochondrial membrane proteins like Voltage-dependent anion channel proteins (VDACs), Fatty acyl-CoA synthetase 1 (ACS-1) and Carnitine palmitoyl-transferase 1 (CPT-1) has been reported to increase their stability while the functional consequence of such acetylation is unknown [71, 72]. Here, we hypothesize that targeting CBP to mitochondria by the fusion protein would result in acetylation and subsequent stabilization of mitochondrial proteins which might provide metabolic advantage for the cancer cell [73, 74].

\section{A}

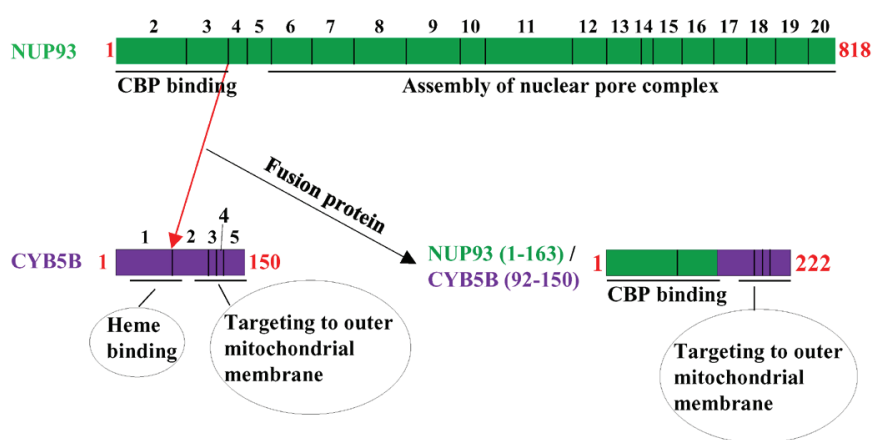

C

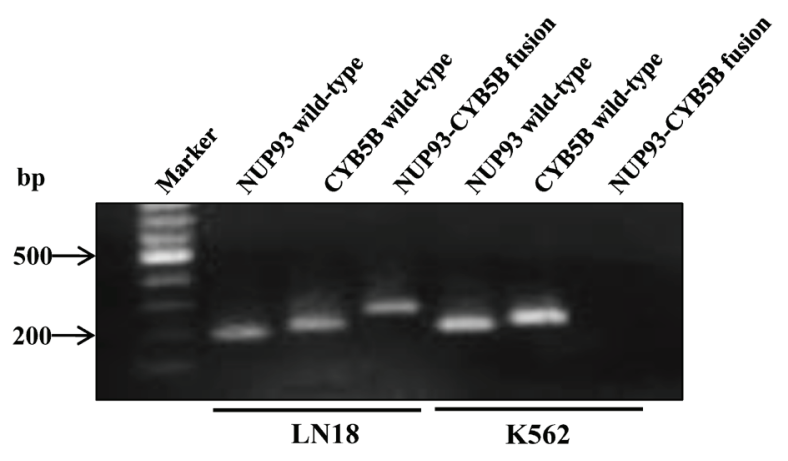

The primer design for the confirmation of fusion product has been given (Figure $8 \mathrm{~B}$ ). The fusion specific primers have been designed such that the forward primer that can amplify the wild-type NUP93 and the reverse primer that can amplify the wild-type CYB5B can be combined to amplify the fusion product such that the amplicon will be of different size than both the wildtype products (Figure 8B). Semi-quantitative analysis of NUP93-CYB5B fusion revealed the presence of the fusion product in LN18 but not in K562, a human leukemic cell line (Figure 8C). Sanger sequencing validation was carried out for the fusion junction (Figure 8D). The exact fusion junction as predicted by PRADA was confirmed in the Sanger sequencing analysis.

\section{GBM specific RNA editing events in the cell lines}

RNA editing is a molecular process by which RNA sequences are altered post-transcriptionally through base conversion or insertion/deletion. In mammals, especially in humans, the most common type of editing changes

B

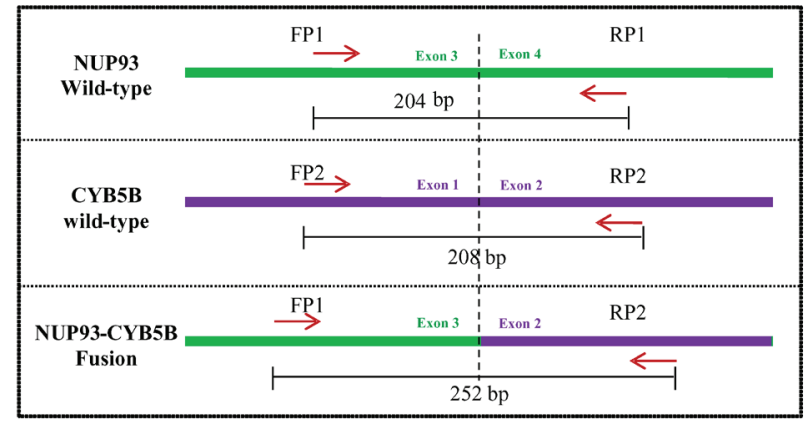

D
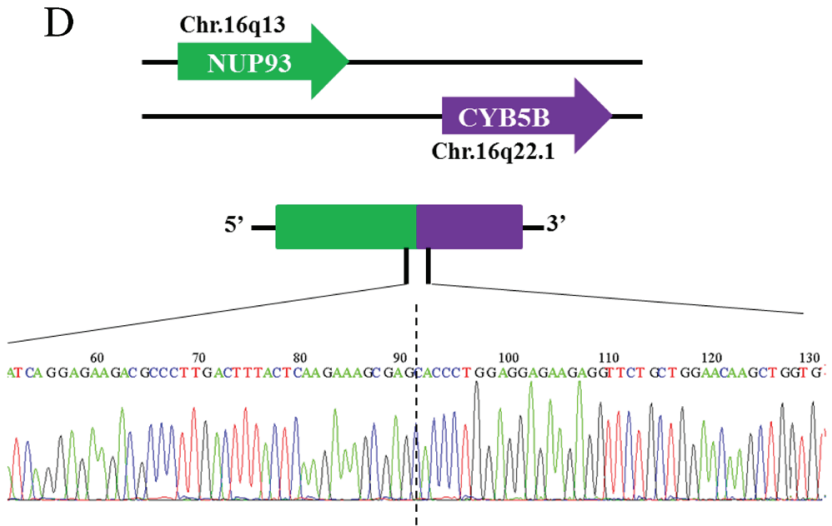

Figure 8: NUP93-CYB5B gene fusion validation. A. Representative image of the protein structure of wild-type NUP93 and CYB5B and NUP93-CYB5B fusion product. The exons representing each section of the protein sequence has been represented by smaller boxes in the protein schematic and exon numbers (black) have been provided on top of the diagram. The amino acid length of each protein sequences have been denoted in red. B. Primer design for detecting wild type and fusion gene for NUP93-CYB5B. FP = Forward primer and RP = Reverse primer. C. Semi-quantitative PCR gel picture of amplicons derived from PCR of wild type NUP93 and CYB5B and NUP93CYB5B fusion products in LN18 and K562. D.. Sanger sequencing chromatogram of the fusion junction region. 
includes $\mathrm{A}$ to $\mathrm{I}$ and $\mathrm{C}$ to $\mathrm{U}$ base modifications (canonical editing events) carried out by ADAR family of enzymes and APOBEC enzymes respectively $[75,76]$. A schematic representation of the pipeline used for detecting potential RNA editing events from RNA-seq data has been provided and the details have been provided in the Materials and Methods section (Supplementary Figure S10). While the average number of editing event per cell line was found to be 18,949 , there were a total of 1,04,904 editing events across the cell lines (Supplementary Figure S10).

Majority of RNA editing events, were found in Alu repeat region $(97.12 \%)$ compared to non-Alu repeat $(0.4 \%)$ and non-repeat regions $(2.49 \%)$ (Figure $9 \mathrm{~A})$. We also found that the majority of the RNA editing events were present in the intronic regions $(61.82 \%)$ followed by intergenic $(34.91 \%)$, UTR $(2.55 \%)$ and exonic regions
$(0.71 \%)$ (Figue $9 \mathrm{~A})$. A total of $75 \%$ of the base changes in the Alu repeat regions were of the types $A>G$ and $T>C$ (complementary base change of $A>G$ ), which are ADAR specific changes (Figure 9B) [77-79]. However, in nonAlu repeat and non-repeat regions, both $\operatorname{ADAR}(\mathrm{A}>\mathrm{G}, \mathrm{T}$ $>\mathrm{C})$ and APOBEC $(\mathrm{C}>\mathrm{T}, \mathrm{G}>\mathrm{A})$ specific RNA editing events were found to occur relatively at higher frequency compared to other editing events (Figure 9B). While the ADAR-specific RNA editing events were seen more in general, it was interesting to note that exonic portions of the genome had higher percentages of APOBEC-specific RNA editing events (Figure 9C). However, other regions like intronic, intergenic and UTR had higher proportion of ADAR-specific RNA editing events (Figure 9C). Low prevalence of Alu repeats in exonic regions explain the reduced ADAR-specific RNA editing events and increased
A

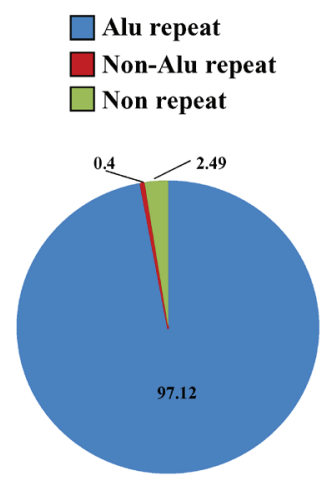

B

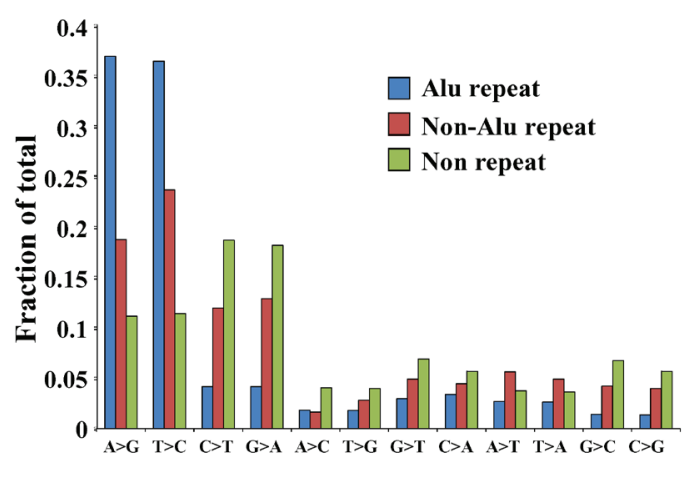

C

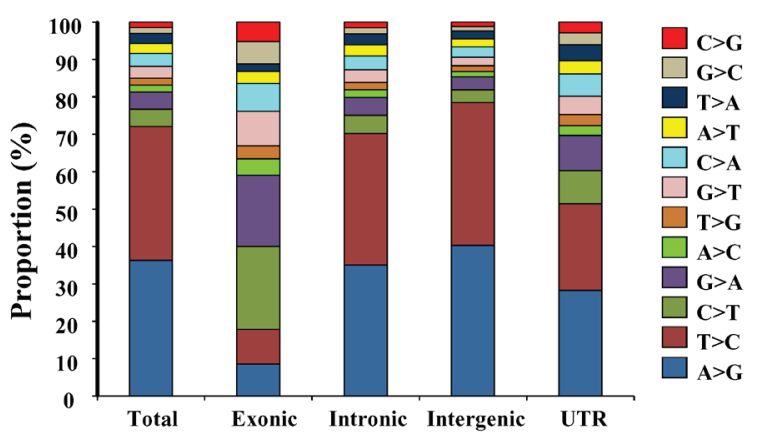

$\mathrm{D}$
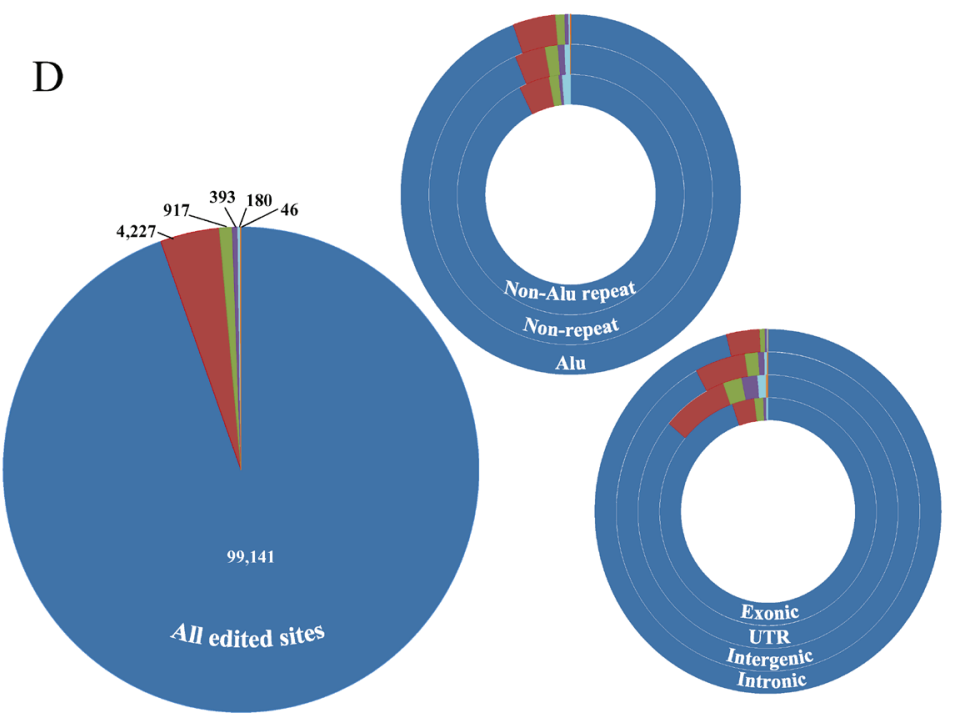

$\square 1 \square 2 \square 3 \square 4 \square 5 \square 6$

Figure 9: Distribution of editing events across the genome. A. Distribution of total editing events in different regions of the genome; left panel: in Alu repeat, non-Alu repeat and non-repeat regions and right panel: in exonic, UTR, intergenic and intronic regions. B. Distribution of different types of editing events in different regions of the genome (Alu repeat, non-Alu repeat and non-repeat). C. Distribution of RNA editing events across different portions of the genome (exonic, intronic, intergenic and UTR). D. Recurrence distribution of editing events in the six cell lines in all the edited sites (big circle) or in non-Alu repeat, non-repeat and Alu repeat regions or in exonic, UTR, intergenic and intronic regions (smaller hollow circles); e.g: 99,141 editing events were unique to one sample, 4,227 were in two samples etc. The color code at the bottom represents in how many cell lines an editing event will be present e.g. 1 represents that editing events are present in one of the six cell lines, 2 represents editing events to be present in two cell lines etc. 
APOBEC-specific RNA editing events. The maximum number of RNA editing events $(94.5 \%)$ were unique to each cell line while a small proportion of them were found in multiple cell lines (Figure 9D). Among Alu repeat, non-Alu repeat and non-repeat regions, the percentage of unique RNA editing events were more or less similar ( 94\%) (Figure 9D). The percentage of recurrent (present in more than one cell line) editing events was maximum in UTR region (13.82\%) followed by intergenic (7.81\%), exonic $(5.36 \%)$ and intronic $(3.86 \%)$ regions (Figure 9D). The list of all the editing events across the cell lines have been tabulated in Supplementary Table 8. Finally, validation by Sanger sequencing was performed and 8 out of 16 selected A to G RNA editing events got validated (Supplementary Figure S7A and S7B).

\section{DISCUSSION}

In this study, we provide a comprehensive characterization of six most widely used GBM cell lines using WES and RNA-seq. Previous studies which characterize glioma cell lines using NGS fall short of comprehensive characterization [10-12]. In the first study, identification of SNVs and structural variations (copy number alterations and gene rearrangments) of one cell line (U87) was carried out from whole genome sequencing (WGS) [10]. In the second study, the authors performed WES of multiple cell lines of different tissue lineages including six GBM cell lines (SF-268, SF-295, SF-539, SNB-19, SNB-75 and U251) and identified indels and cancer-specific SNVs to understand the pharmacogenomic correlations between specific variants and sensitivity to various anti-cancer drugs [11]. In the third study, characterization of 675 human cancer cell lines was carried out to identify SNVs and gene fusion events from RNA sequencing data [12]. This study included 22 cell lines which were glioma-derived of which three cell lines (T98G, LN229 and LN18) are characterized in the current study. In contrast, we have carried out both WES and RNA-seq of six most commonly used GBM cell lines (T98G, LN229, LN18, U87, U343 and U373). Further, an in-depth data analysis was performed to find overall genetic alterations that included SNVs, indels, transcriptome changes, gene fusions and RNA editing events. Moreover, we have validated various genetic alterations (SNVs, indels, gene fusion and RNA editing events) through Sanger sequencing and quantitative PCR based methods.

We particularly focus on the genetic alterations in cell lines which have potential roles in carcinogenesis. From the total SNV data, we have carried out analysis to unearth cancer-specific alterations. Further, we compared the mutation spectrum of GBM cell lines with that of GBM tissue samples reported by other groups $[8,9]$ and this revealed various genes altered in GBM scenario to be altered in these cell lines also. Although Klijn et al. have uncovered SNVs from RNA-seq data, analysis of SNVs from whole exome sequencing data will be more comprehensive as it will cover vast majority of all the protein coding genes. SNV data of genes not expressed or expressed at low levels in RNA copy numbers will be missed out from RNA-seq data. We have also carried out indel analysis from WES. Functional importance of genes altered by mutation like TP53, MLL3, BRCA2, NF1 etc. has been investigated. Important emphasis has also been given to hTERT promoter mutation status through Sanger sequencing as this particular mutation is predominant among GBM patients. We have checked the gene expression levels in GBM cell lines with respect to normal brain samples and we have found good concordance with GBM tumor tissue microarray data. Moreover, we also analyzed both RNA-seq and WES data to find out potential RNA editing events. Further, we compared these editing events with normal brain editome data to find out editing events occurring in diseased condition. To our knowledge, such a comprehensive study of genetic alterations in GBM cell lines has not been carried out till now.

Among indels and cancer-specific SNVs reported previously to be present in GBM tumor tissues [8,9], the most frequent alterations were observed in TP53, PTEN, TCHH and MLL3. Further, mutations in EGFR, NF1 and PDGFRA were found in some of the cell lines. Chromatin modifier genes like MLL3, ATRX, MLL4, SETD2 and SRCAP were seen to be mutated. The six GBM cell lines were seen to harbor mutations in 19 DNA repair related genes. However, none of the genes in BER or MMR pathways were observed to be mutated in any of the cell lines. Global gene expression profiling followed by pathway analysis of p53 mutant vs p 53 wild-type cell lines revealed p53 regulated processes like cell differentiation, neuron development, cell adhesion, cell proliferation and apoptosis to be significantly enriched. Interestingly, none of the cell lines showed mutation in IDH1 gene. Our study also revealed that U87, T98G, LN229, U343, U251, and U373 cell lines harbor promoter activating mutations in the hTERT genes with a concomitant up regulation of hTERT transcript levels. No mutation was found in LN18 and SVG and hTERT mRNA levels were also found to be low in these cell lines. A comparison of the differentially regulated genes in the cell lines with that of GBM tumor transcriptome data derived from TCGA gave a concordance of $\sim 73 \%$ suggesting that the transcriptomic make up of these cell lines closely resemble that of GBM tumor tissues. A total of over three hundred gene fusion events were identified in the six cell lines using three different tools and five such fusions came up to be common in all three tools. Two gene fusions, NUP93CYB5B and STX17-NR4A3, were in-frame fusions both of which were present in LN18 cell line. Validation of NUP93-CYB5B fusion using Sanger sequencing revealed the exact fusion junction as predicted by PRADA. The targeting of CBP to the mitochondrial outer membrane 
by NUP93-CYB5B fusion protein is likely to result in the acetylation of proteins like VDACs, ACS-1 and CPT-1 which may provide metabolic advantage to the cells. While ACS1 and CPT1 are involved in fatty acid metabolism and transport into mitochondria respectively, VDACs help in ATP transport out of mitochondria [73, 80]. Further, $\beta$-oxidation of fatty acids in the mitochondria results in increased energy production in the cancer cells with the resultant increased proliferation [73]. Indeed, inhibition of fatty acid synthesis or its transport into mitochondria results in growth inhibition and increased apoptosis $[73,74]$. Hence, we predict that CBP-mediated acetylation of these proteins with resultant stabilization is likely to provide survival advantage to the cancer cells. Further, RNA editome analysis revealed numerous RNA editing events across the cell lines. As observed by others, we found majority of RNA editing events in Alu repeat region present both in intronic and intergenic regions, while its significance is not known yet [81]. We also found significant RNA editing events in the UTR region, in particular 3'UTR, which is likely to alter miRNA binding leading to the alteration of the gene expression pattern which may contribute to transformation and other cancer cell properties [82].

Thus, a comprehensive alteration landscape that includes cancer specific SNVs, indels, transcriptome profile, gene fusions and RNA editing events is generated from whole exome and RNA sequencing data for six GBM cell lines. Since these cell lines are routinely used for in vitro and in vivo studies by glioma biologists, our study would be of great help to the scientific community.

\section{MATERIALS AND METHODS}

\section{Cell lines used}

The cell lines used are glioblastoma (GBM) cell lines U87, U343, U251, U373, LN18, LN229 and T98G and immortalized human astrocytic cell lines SVG [83] and IHA (NHA-E6/E7-hTERT) [84]. U343, LN18, IHA and SVG were obtained from the laboratory of Dr. A. Guha, University of Toronto, Canada. U87, T98G, U251, LN229 and U373 were obtained from Sigma Aldrich (Saint Louis, Missouri, USA). All cell lines were cultured in Dulbecco's Modified Eagles' Medium containing 10\% Fetal Bovine Serum at $37^{\circ} \mathrm{C}$ and $5 \% \mathrm{CO}_{2}$.

\section{DNA isolation, quantification and library preparation for whole exome sequencing}

DNA was isolated from 5 million cells for each cell line using Qiagen DNA Minikit (Catalog no. 51306) for obtaining good quality protein and RNA free genomic DNA. The concentration of DNA was determined using
Quant-iT ${ }^{\text {TM }}$ PicoGreen ${ }^{\circledR}$ dsDNA Assay Kit (Catalog no. P11496) where the standard curve was obtained from increasing dilutions of lambda DNA (Stock = $500 \mathrm{ng} / \mu \mathrm{l})$. DNA library was prepared using TruSeq Library Preparation kit (Catalog no. RS-122-2001) as per manufacturer's guidelines. DNA was sheared using Covaris sonicator (S220) and the library was prepared. The obtained DNA library was quantified using Agilent's Biolanalyzer. Next, DNA libraries (one for each sample) were taken in batches of six and pooled together for exome enrichment using Truseq Exome Enrichment kit (Catalog no. FC-121-1008). Finally the exome libraries were quantified by real-time qPCR using Illumina adapter specific primers for library quantification.

\section{RNA isolation, quantification and library preparation for whole RNA sequencing}

Cells were harvested using Tri Reagent ${ }^{\circledR}$ (Sigma, Catalog no. T9424) and RNA were extracted using the standard Chloroform-Isopropanol method. The RNA samples were checked for quality and quantity using Agilent's Bioanalyzer. The library for sequencing was prepared using TrueSeq RNA sample preparation kit as per the manufacturer's guidelines (Catalog no. RS-1222001). The library was then quantified using Agilent's Bioanalyzer as well as real-time qPCR.

\section{Cluster generation and sequencing}

10 picomoles of each pooled exome or RNA library was taken, the strands were denatured and finally subjected to cluster generation on the flow-cell in the c-Bot system using TruSeq PE Cluster kit (Catalog no. PE-401-3001). The flow cell was finally subjected to two rounds of sequencing (for Read1 and $\operatorname{Read} 2$ ) and the results were obtained as intensity files. Sequencing was conducted on Illumina HiScanSQ using Truseq SBS V3 technology for 100 and 50 base pair paired-end reads for exome and RNA sequencing respectively (Catalog nos. FC-401-3001 and FC-401-3002 respectively).

\section{cDNA conversion}

For cDNA conversion, $2 \mu \mathrm{g}$ good quality RNA was used per reaction. Applied Biosystems ${ }^{\mathrm{TM}}$ High Capacity cDNA Reverse Transcription kit (Part no. 4368813) was used. The cDNA strand synthesis was carried out in Biorad S1000 TM Thermal Cycler.

\section{PCR amplification and Sanger sequencing}

Genomic DNA or cDNA was taken as template for PCR amplification. Thermo Scientific's DyNAmo 
(Catalog no. F-416) reagent was used as amplification buffer along with primer of interest. Biorad S1000'TM Thermal Cycler was used for PCR amplification.

Primer sequences have been provided in Supplementary Table S9. A schematic representation of the PCR strategy used for amplification of the promoter region of hTERT has been given (Supplementary Figure S8).

\section{Real-time qPCR quantification}

Thermo Scientific's DyNAmo (Catalog no. F-416) reagent was used for this purpose with cDNA from good quality RNA used as template. Applied Biosystems ${ }^{\mathrm{TM}}$ 7900HT Fast Real-Time PCR system was used. GAPDH was used as internal control. Primer sequences have been provided in Supplementary Table S9.

\section{Data analysis pipelines}

\section{Whole exome sequencing analysis}

We have carried out 100 bp paired-end sequencing for this purpose.

\section{Alignment and Recalibration}

The sequencing output in the form of base intensity files was converted to fastq format and subsequently demultiplexed using bcl2fastq [85]. Next, BWA or Burrows Wheeler Aligner version 0.6.2 was used to align the reads to the human reference genome hg19 i.e., Human Genome Reference Consortium build 37 (GRCh37) [86]. Post-alignment, we obtained the '.sam' file which was converted to binary format or '.bam' files using Samtools 0.1.18 [87]. For co-ordinate sorting and duplicate removal, Picard 1.73 was used [88]. Read re-alignment, required for properly calling indels, was done using GATK 2.72 module IndelRealigner [89]. The same tool's module called BaseRecalibrator was used for base recalibration.

\section{Variant Calling}

The detection of Single nucleotide variants (SNVs) and indels (small insertions/deletions) was carried out using GATK's module called UnifiedGenotyper [89]. Finally, variant annotation was done using Oncotator v.4.2.2 [17].

\section{Filtering for Cancer-specific SNVs}

To filter out cancer-specific SNVs from total SNVs, the SNVs obtained from GATK UnifiedGenotyper was passed through stringent filteration steps (Supplementary Figure S9). SNVs with ESP6500 frequency $\geq 0.00009$ were removed as SNVs having no disease consequences [90]. Similarly, in the next step, SNVs with 1000 genomes database frequency $\geq 0.0005$ were eliminated [91]. The SNVs obtained at this step were divided into those reported in COSMIC database versus those not reported in the same [18]. The SNVs present in COSMIC were considered as single nucleotide changes that may have possible role in carcinogenesis and hence cancerspecific SNVs. Those SNVs not present in COSMIC were compared with the SNVs obtained from GBM TCGA exome sequencing data $[8,9]$. Those SNVs present in the TCGA data were taken to be cancer-specific changes while SNVs not present in the above database were taken up for the following steps of filtration. SNVs with data set frequency, i.e. frequency of occurrence among the samples tested, $\geq 0.5$ were eliminated. Finally the above SNVs were compared with dbSNP build 137 to remove potential single nucleotide polymorphisms [92]. Hence, all mutations obtained from the previous step, those obtained as present in COSMIC database and those which came up as already reported GBM-specific changes as per TCGA were combined to form the cancer-specific SNVs set. The rest of the SNVs were considered as non-specific.

\section{RNA sequencing analysis}

We have carried out 50 bp paired-end sequencing for this purpose.

\section{Transcriptome analysis}

The whole RNA sequencing data was aligned using PRADA tool [65]. Duplicate removal was carried out using Picard 1.73 [88]. The RNA-seq reads were counted over gene exons using HtSeq [93]. Genes were annotated as per Gencode Version 19 annotation file [55]. We used the DESeq size factor correction to account for differences in sequencing depth between the samples. For differentially expressed gene identification between GBM cell lines compared to normal brain tissue samples (from TCGA), we used DESeq with p-adjusted cut-off of 0.05 [94].

\section{Gene fusion analysis}

Analysis of potential gene fusions in each cell line from RNA sequencing data was carried out using three different tools - PRADA, FusionMap and TopHat-Fusion. 1) PRADA: Pipeline for RNA sequencing Data Analysis [65]. PRADA aligns RNA sequencing reads to a composite reference database comprising of whole genome reference sequence (hg19) and reference transcriptome sequence (Ensemb164) using BWA. To filter out potential gene fusions, the following criteria were used- -mm 3 -junL 40 -minmapq 30. 2) FusionMap: RNA-seq reads for each cell line was aligned using PRADA-Preprocess-bi tool and the aligned reads were then fed into FusionMap [63]. The following parameters were used for fusion calling MinimalFusionAlignmentLength $=25$ FusionReportCutoff $=1$ NonCanonicalSpliceJunctionPenalty $=2$ MinimalHit $=2$ MinimalRescuedReadNumber $=1.3$ ) TopHat-Fusion: The RNA-seq data was mapped using TopHat 2 with the 
following options -r 0 -p 14 -no-coverage-search -matestd-dev 80 -max-intron-length 100000 -fusion-min-dist 100000 -fusion-anchor-length 13 -fusion-search -keepfasta-order -bowtie1. Using the mapped RNA-seq data, fusion transcript candidates were filtered by tophat-fusionpost [64].

\section{RNA editing events}

RNA-seq data from each cell line was used to identify RNA editing events following a rigorous and robust pipeline (Supplementary Figure S10) [95, 96]. The RNA-seq reads were aligned to reference genome (hg19) and transcriptome (Ensembl64) using PRADAPreprocess-bi tool. Next, variants were called using GATK's UnifiedGenotyper with options stand_call conf of 0 and stand_emit_conf of 0 [89]. The total variants obtained were then filtered to remove potential polymorphisms by comparing with dbSNP [92], 1000 genomes database [91] and ESP6500 [90]. First six bases of each read were discarded to remove artificial mismatches caused by random-hexamer priming. The editing events present in Alu regions were separated out. The editing events present in non-Alu regions were then subjected to further steps to filter out spurious changes1) each editing event were to be represented by atleast 3 reads containing altered nucleotide with a minimum frequency of altered nucleotide being $0.1 ; 2$ ) any site present in simple repeats were removed; 3) any candidate change present within 4 bps of known splice junction were removed; 4) sites present in homopolymer runs of $\geq 5$ bps were removed; and, 5) sites located in regions having high similarity to sequences present in other parts of the genome (found out using BLAT) were removed. Editing events present in non-Alu regions along with those found in the Alu regions were compared with normal brain editome data to eliminate editing events which have no carcinogenic consequences [96]. Events obtained from above step were compared with SNVs obtained from whole exome sequencing data of each corresponding sample to remove RNA editing events that are wrongly called due to presence of sample-specific genetic variations. This gives GBM cell-line specific RNA editing events.

\section{Abbreviations}

GBM-glioblastoma, SNP-Single Nucleotide Polymorphism, SNV-Single Nucleotide Variation, indels - insertions and deletions, NGS- Next Generation Sequencing, WES- Whole Exome Sequencing, RNAseq - RNA Sequencing,TCGA - The Cancer Genome Atlas, CCLE- Cancer Cell Line Encyclopedia, COSMIC - Catalogue Of Somatic Mutations In Cancer, IHA Immortalized Human Astrocytes.

\section{ACKNOWLEDGMENTS}

The results published here are in whole or part based upon data generated by The Cancer Genome Atlas pilot project established by the NCI and NHGRI. Information about TCGA and the investigators and institutions who constitute the TCGA research network can be found at http://cancergenome.nih.gov/. JP and VP thank DBT, Government of India for financial support. This study was supported by a grant from DBT, government of India. The NGS facility, Indian Institute of Science is acknowledged for DNA and RNA sequencing. Infrastructural support by funding from DBT, DST and UGC to MCB is acknowledged. KS is a J. C. Bose Fellow of the Department of Science and Technology. Prof. Chitra Sarkar (AIIMS), Sujaya Srinivasan and Arjun Arkal Rao are acknowledged.

\section{CONFLICTS OF INTEREST}

There is no conflict of interest

\section{REFERENCES}

1. MLJ A. Malignant Cerebral Glioma (Neurosurgical Topics, 2). (Neurosurgical Topics, 2). 1990.

2. Ohgaki $\mathrm{H}$ and Kleihues $\mathrm{P}$. The definition of primary and secondary glioblastoma. Clinical cancer research : an official journal of the American Association for Cancer Research. 2013; 19:764-772.

3. Stupp R, Hegi ME, Mason WP, van den Bent MJ, Taphoorn MJ, Janzer RC, Ludwin SK, Allgeier A, Fisher B, Belanger K, Hau P, Brandes AA, Gijtenbeek J, Marosi C, Vecht CJ, Mokhtari K, et al. Effects of radiotherapy with concomitant and adjuvant temozolomide versus radiotherapy alone on survival in glioblastoma in a randomised phase III study: 5-year analysis of the EORTC-NCIC trial. Lancet Oncol. 2009; 10:459-466.

4. Furnari FB, Fenton T, Bachoo RM, Mukasa A, Stommel JM, Stegh A, Hahn WC, Ligon KL, Louis DN, Brennan C, Chin L, DePinho RA and Cavenee WK. Malignant astrocytic glioma: genetics, biology, and paths to treatment. Genes \& development. 2007; 21:2683-2710.

5. Cancer Genome Atlas Research N. Comprehensive genomic characterization defines human glioblastoma genes and core pathways. Nature. 2008; 455:1061-1068.

6. Meyerson M, Gabriel S and Getz G. Advances in understanding cancer genomes through second-generation sequencing. Nature reviews Genetics. 2010; 11:685-696.

7. Robison K. Application of second-generation sequencing to cancer genomics. Briefings in bioinformatics. 2010; 11:524534.

8. Frattini V, Trifonov V, Chan JM, Castano A, Lia M, Abate F, Keir ST, Ji AX, Zoppoli P, Niola F, Danussi C, 
Dolgalev I, Porrati P, Pellegatta S, Heguy A, Gupta G, et al. The integrated landscape of driver genomic alterations in glioblastoma. Nat Genet. 2013; 45:1141-1149.

9. Brennan CW, Verhaak RG, McKenna A, Campos B, Noushmehr H, Salama SR, Zheng S, Chakravarty D, Sanborn JZ, Berman SH, Beroukhim R, Bernard B, Wu CJ, Genovese G, Shmulevich I, Barnholtz-Sloan J, et al. The somatic genomic landscape of glioblastoma. Cell. 2013; 155:462-477.

10. Clark MJ, Homer N, O'Connor BD, Chen Z, Eskin A, Lee H, Merriman B and Nelson SF. U87MG decoded: the genomic sequence of a cytogenetically aberrant human cancer cell line. PLoS genetics. 2010; 6:e1000832.

11. Abaan OD, Polley EC, Davis SR, Zhu YJ, Bilke S, Walker RL, Pineda M, Gindin Y, Jiang Y, Reinhold WC, Holbeck SL, Simon RM, Doroshow JH, Pommier Y and Meltzer PS. The exomes of the NCI-60 panel: a genomic resource for cancer biology and systems pharmacology. Cancer Res. 2013; 73:4372-4382.

12. Klijn C, Durinck S, Stawiski EW, Haverty PM, Jiang Z, Liu H, Degenhardt J, Mayba O, Gnad F, Liu J, Pau G, Reeder J, Cao Y, Mukhyala K, Selvaraj SK, Yu M, et al. A comprehensive transcriptional portrait of human cancer cell lines. Nature biotechnology. 2015; 33:306-312.

13. http://www.illumina.com/content/dam/illumina-marketing/ documents/products/technotes/technote_snp_caller_ sequencing.pdf.

14. http://www.illumina.com/content/dam/illumina-marketing/ documents/products/datasheets/datasheet-truseq-rna-access. pdf.

15. Clark MJ, Chen R, Lam HY, Karczewski KJ, Chen R, Euskirchen G, Butte AJ and Snyder M. Performance comparison of exome DNA sequencing technologies. Nature biotechnology. 2011; 29:908-914.

16. Gu X and Li WH. The size distribution of insertions and deletions in human and rodent pseudogenes suggests the logarithmic gap penalty for sequence alignment. Journal of molecular evolution. 1995; 40:464-473.

17. http://www.broadinstitute.org/oncotator/.

18. Forbes SA, Beare D, Gunasekaran P, Leung K, Bindal N, Boutselakis H, Ding M, Bamford S, Cole C, Ward S, Kok CY, Jia M, De T, Teague JW, Stratton MR, McDermott $\mathrm{U}$, et al. COSMIC: exploring the world's knowledge of somatic mutations in human cancer. Nucleic acids research. 2015; 43:D805-811.

19. Barretina J, Caponigro G, Stransky N, Venkatesan K, Margolin AA, Kim S, Wilson CJ, Lehar J, Kryukov GV, Sonkin D, Reddy A, Liu M, Murray L, Berger MF, Monahan JE, Morais P, et al. The Cancer Cell Line Encyclopedia enables predictive modelling of anticancer drug sensitivity. Nature. 2012; 483:603-607.

20. Martinez R, Schackert HK, Plaschke J, Baretton G, Appelt $\mathrm{H}$ and Schackert G. Molecular mechanisms associated with chromosomal and microsatellite instability in sporadic glioblastoma multiforme. Oncology. 2004; 66:395-403.

21. Adzhubei I, Jordan DM and Sunyaev SR. Predicting functional effect of human missense mutations using PolyPhen-2. Curr Protoc Hum Genet. 2013; Chapter 7:Unit7 20.

22. Kumar P, Henikoff S and Ng PC. Predicting the effects of coding non-synonymous variants on protein function using the SIFT algorithm. Nat Protoc. 2009; 4:1073-1081.

23. Poullet $\mathrm{P}$, Lin $\mathrm{B}$, Esson $\mathrm{K}$ and Tamanoi F. Functional significance of lysine 1423 of neurofibromin and characterization of a second site suppressor which rescues mutations at this residue and suppresses RAS2Val-19activated phenotypes. Mol Cell Biol. 1994; 14:815-821.

24. Parsons DW, Li M, Zhang X, Jones S, Leary RJ, Lin JC, Boca SM, Carter H, Samayoa J, Bettegowda C, Gallia GL, Jallo GI, Binder ZA, Nikolsky Y, Hartigan J, Smith DR, et al. The genetic landscape of the childhood cancer medulloblastoma. Science. 2011; 331:435-439.

25. Herz HM, Hu D and Shilatifard A. Enhancer malfunction in cancer. Molecular cell. 2014; 53:859-866.

26. UniProt C. UniProt: a hub for protein information. Nucleic acids research. 2015; 43:D204-212.

27. Tanaka A, Weinel S, Nagy N, O’Driscoll M, Lai-Cheong JE, Kulp-Shorten CL, Knable A, Carpenter G, Fisher SA, Hiragun M, Yanase Y, Hide M, Callen J and McGrath JA. Germline mutation in ATR in autosomal- dominant oropharyngeal cancer syndrome. American journal of human genetics. 2012; 90:511-517.

28. Takayama K, Salazar EP, Lehmann A, Stefanini M, Thompson LH and Weber CA. Defects in the DNA repair and transcription gene ERCC2 in the cancer-prone disorder xeroderma pigmentosum group D. Cancer research. 1995; 55:5656-5663.

29. Huang da W, Sherman BT and Lempicki RA. Systematic and integrative analysis of large gene lists using DAVID bioinformatics resources. Nat Protoc. 2009; 4:44-57.

30. Huang DW, Sherman BT, Tan Q, Kir J, Liu D, Bryant D, Guo Y, Stephens R, Baseler MW, Lane HC and Lempicki RA. DAVID Bioinformatics Resources: expanded annotation database and novel algorithms to better extract biology from large gene lists. Nucleic Acids Res. 2007; 35:W169-175.

31. Stiewe T. The p53 family in differentiation and tumorigenesis. Nat Rev Cancer. 2007; 7:165-168.

32. Molchadsky A, Rivlin N, Brosh R, Rotter V and Sarig R. p53 is balancing development, differentiation and dedifferentiation to assure cancer prevention. Carcinogenesis. 2010; 31:1501-1508.

33. Qin H, Yu T, Qing T, Liu Y, Zhao Y, Cai J, Li J, Song Z, Qu X, Zhou P, Wu J, Ding M and Deng H. Regulation of apoptosis and differentiation by p53 in human embryonic stem cells. J Biol Chem. 2007; 282:5842-5852.

34. Tedeschi A and Di Giovanni S. The non-apoptotic role of p53 in neuronal biology: enlightening the dark side of the 
moon. EMBO Rep. 2009; 10:576-583.

35. Ferreira A and Kosik KS. Accelerated neuronal differentiation induced by p53 suppression. J Cell Sci. 1996; 109 :1509-1516.

36. Yang X, Klein R, Tian X, Cheng HT, Kopan R and Shen J. Notch activation induces apoptosis in neural progenitor cells through a p53-dependent pathway. Dev Biol. 2004; 269:81-94.

37. Yeudall WA, Wrighton KH and Deb S. Mutant p53 in cell adhesion and motility. Methods in molecular biology. 2013; 962:135-146.

38. Anaganti S, Fernandez-Cuesta L, Langerod A, Hainaut P and Olivier M. p53-Dependent repression of focal adhesion kinase in response to estradiol in breast cancer cell-lines. Cancer Lett. 2011; 300:215-224.

39. Golubovskaya VM, Ho B, Zheng M, Magis A, Ostrov D, Morrison $\mathrm{C}$ and Cance WG. Disruption of focal adhesion kinase and p53 interaction with small molecule compound $\mathrm{R} 2$ reactivated $\mathrm{p} 53$ and blocked tumor growth. BMC Cancer. 2013; 13:342.

40. Lee JG, Ahn JH, Jin Kim T, Ho Lee J and Choi JH. Mutant p53 promotes ovarian cancer cell adhesion to mesothelial cells via integrin beta4 and Akt signals. Sci Rep. 2015; 5:12642.

41. Wawryk-Gawda E, Chylinska-Wrzos P, Lis-Sochocka M, Chlapek K, Bulak K, Jedrych M and Jodlowska-Jedrych B. P53 protein in proliferation, repair and apoptosis of cells. Protoplasma. 2014; 251:525-533.

42. Mollereau B and Ma D. The p53 control of apoptosis and proliferation: lessons from Drosophila. Apoptosis. 2014; 19:1421-1429.

43. Mercer WE, Avignolo C and Baserga R. Role of the p53 protein in cell proliferation as studied by microinjection of monoclonal antibodies. Mol Cell Biol. 1984; 4:276-281.

44. Schreiber M, Kolbus A, Piu F, Szabowski A, MohleSteinlein U, Tian J, Karin M, Angel P and Wagner EF. Control of cell cycle progression by c-Jun is p53 dependent. Genes Dev. 1999; 13:607-619.

45. Amaral JD, Xavier JM, Steer CJ and Rodrigues CM. The role of p53 in apoptosis. Discov Med. 2010; 9:145-152.

46. Haupt S, Berger M, Goldberg Z and Haupt Y. Apoptosis the p53 network. J Cell Sci. 2003; 116:4077-4085.

47. Amundson SA, Myers TG and Fornace AJ, Jr. Roles for p53 in growth arrest and apoptosis: putting on the brakes after genotoxic stress. Oncogene. 1998; 17:3287-3299.

48. Parsons DW, Jones S, Zhang X, Lin JC, Leary RJ, Angenendt P, Mankoo P, Carter H, Siu IM, Gallia GL, Olivi A, McLendon R, Rasheed BA, Keir S, Nikolskaya T, Nikolsky Y, et al. An integrated genomic analysis of human glioblastoma multiforme. Science. 2008; 321:1807-1812.

49. Watanabe $\mathrm{T}$, Nobusawa $\mathrm{S}$, Kleihues $\mathrm{P}$ and Ohgaki H. IDH1 mutations are early events in the development of astrocytomas and oligodendrogliomas. The American journal of pathology. 2009; 174:1149-1153.
50. Kato Y, Jin G, Kuan CT, McLendon RE, Yan H and Bigner DD. A monoclonal antibody IMab-1 specifically recognizes IDH1R132H, the most common glioma-derived mutation. Biochemical and biophysical research communications. 2009; 390:547-551.

51. Cohen AL, Holmen SL and Colman H. IDH1 and IDH2 mutations in gliomas. Current neurology and neuroscience reports. 2013; 13:345.

52. Killela PJ, Reitman ZJ, Jiao Y, Bettegowda C, Agrawal N, Diaz LA, Jr., Friedman AH, Friedman H, Gallia GL, Giovanella BC, Grollman AP, He TC, He Y, Hruban RH, Jallo GI, Mandahl N, et al. TERT promoter mutations occur frequently in gliomas and a subset of tumors derived from cells with low rates of self-renewal. Proceedings of the National Academy of Sciences of the United States of America. 2013; 110:6021-6026.

53. Nonoguchi N, Ohta T, Oh JE, Kim YH, Kleihues P and Ohgaki H. TERT promoter mutations in primary and secondary glioblastomas. Acta neuropathologica. 2013; 126:931-937.

54. Arita H, Narita Y, Fukushima S, Tateishi K, Matsushita Y, Yoshida A, Miyakita Y, Ohno M, Collins VP, Kawahara $\mathrm{N}$, Shibui $\mathrm{S}$ and Ichimura K. Upregulating mutations in the TERT promoter commonly occur in adult malignant gliomas and are strongly associated with total 1p19q loss. Acta neuropathologica. 2013; 126:267-276.

55. http://www.gencodegenes.org/releases/19.html.

56. Nowell PC. Discovery of the Philadelphia chromosome: a personal perspective. J Clin Invest. 2007; 117:2033-2035.

57. Soda M, Choi YL, Enomoto M, Takada S, Yamashita Y, Ishikawa S, Fujiwara S, Watanabe H, Kurashina K, Hatanaka H, Bando M, Ohno S, Ishikawa Y, Aburatani H, Niki T, Sohara Y, et al. Identification of the transforming EML4-ALK fusion gene in non-small-cell lung cancer. Nature. 2007; 448:561-566.

58. Tomlins SA, Rhodes DR, Perner S, Dhanasekaran SM, Mehra R, Sun XW, Varambally S, Cao X, Tchinda J, Kuefer R, Lee C, Montie JE, Shah RB, Pienta KJ, Rubin MA and Chinnaiyan AM. Recurrent fusion of TMPRSS2 and ETS transcription factor genes in prostate cancer. Science. 2005; 310:644-648.

59. Ju YS, Lee WC, Shin JY, Lee S, Bleazard T, Won JK, Kim YT, Kim JI, Kang JH and Seo JS. A transforming KIF5B and RET gene fusion in lung adenocarcinoma revealed from whole-genome and transcriptome sequencing. Genome research. 2012; 22:436-445.

60. Singh D, Chan JM, Zoppoli P, Niola F, Sullivan R, Castano A, Liu EM, Reichel J, Porrati P, Pellegatta S, Qiu K, Gao Z, Ceccarelli M, Riccardi R, Brat DJ, Guha A, et al. Transforming fusions of FGFR and TACC genes in human glioblastoma. Science. 2012; 337:1231-1235.

61. Shah N, Lankerovich M, Lee H, Yoon JG, Schroeder B and Foltz G. Exploration of the gene fusion landscape of glioblastoma using transcriptome sequencing and copy 
number data. BMC genomics. 2013; 14:818.

62. Kim J, Lee Y, Cho HJ, Lee YE, An J, Cho GH, Ko YH, Joo KM and Nam DH. NTRK1 fusion in glioblastoma multiforme. PLoS One. 2014; 9:e91940.

63. Ge H, Liu K, Juan T, Fang F, Newman M and Hoeck W. FusionMap: detecting fusion genes from next-generation sequencing data at base-pair resolution. Bioinformatics. 2011; 27:1922-1928.

64. Kim D and Salzberg SL. TopHat-Fusion: an algorithm for discovery of novel fusion transcripts. Genome biology. 2011; 12:R72.

65. Torres-Garcia W, Zheng S, Sivachenko A, Vegesna R, Wang Q, Yao R, Berger MF, Weinstein JN, Getz G and Verhaak RG. PRADA: pipeline for RNA sequencing data analysis. Bioinformatics. 2014; 30:2224-2226.

66. Sachdev R, Sieverding C, Flotenmeyer M and Antonin W. The C-terminal domain of Nup93 is essential for assembly of the structural backbone of nuclear pore complexes. Molecular biology of the cell. 2012; 23:740-749.

67. Ryan CM, Harries JC, Kindle KB, Collins HM and Heery DM. Functional interaction of CREB binding protein (CBP) with nuclear transport proteins and modulation by HDAC inhibitors. Cell cycle. 2006; 5:2146-2152.

68. De Silvestris M, D'Arrigo A and Borgese N. The targeting information of the mitochondrial outer membrane isoform of cytochrome b5 is contained within the carboxyl-terminal region. FEBS letters. 1995; 370:69-74.

69. Ghanta S, Grossmann RE and Brenner C. Mitochondrial protein acetylation as a cell-intrinsic, evolutionary driver of fat storage: chemical and metabolic logic of acetyllysine modifications. Critical reviews in biochemistry and molecular biology. 2013; 48:561-574.

70. Scott I, Webster BR, Li JH and Sack MN. Identification of a molecular component of the mitochondrial acetyltransferase programme: a novel role for GCN5L1. The Biochemical journal. 2012; 443:655-661.

71. Distler AM, Kerner J and Hoppel CL. Post-translational modifications of rat liver mitochondrial outer membrane proteins identified by mass spectrometry. Biochimica et biophysica acta. 2007; 1774:628-636.

72. Distler AM, Kerner J and Hoppel CL. Proteomics of mitochondrial inner and outer membranes. Proteomics. 2008; 8:4066-4082.

73. Currie E, Schulze A, Zechner R, Walther TC and Farese RV, Jr. Cellular fatty acid metabolism and cancer. Cell metabolism. 2013; 18:153-161.

74. Samudio I, Harmancey R, Fiegl M, Kantarjian H, Konopleva M, Korchin B, Kaluarachchi K, Bornmann W, Duvvuri S, Taegtmeyer H and Andreeff M. Pharmacologic inhibition of fatty acid oxidation sensitizes human leukemia cells to apoptosis induction. The Journal of clinical investigation. 2010; 120:142-156.

75. Gott JM and Emeson RB. Functions and mechanisms of RNA editing. Annual review of genetics. 2000; 34:499-531.
76. Rosenberg BR, Hamilton CE, Mwangi MM, Dewell S and Papavasiliou FN. Transcriptome-wide sequencing reveals numerous APOBEC1 mRNA-editing targets in transcript 3' UTRs. Nature structural \& molecular biology. 2011; 18:230-236.

77. Wulff BE, Sakurai M and Nishikura K. Elucidating the inosinome: global approaches to adenosine-to-inosine RNA editing. Nature reviews Genetics. 2011; 12:81-85.

78. Mo F, Wyatt AW, Sun Y, Brahmbhatt S, McConeghy BJ, Wu C, Wang Y, Gleave ME, Volik SV and Collins CC. Systematic identification and characterization of RNA editing in prostate tumors. PLoS One. 2014; 9:e101431.

79. Levanon EY, Eisenberg E, Yelin R, Nemzer S, Hallegger M, Shemesh R, Fligelman ZY, Shoshan A, Pollock SR, Sztybel D, Olshansky M, Rechavi G and Jantsch MF. Systematic identification of abundant A-to-I editing sites in the human transcriptome. Nature biotechnology. 2004; 22:1001-1005.

80. Shoshan-Barmatz V, Ben-Hail D, Admoni L, Krelin Y and Tripathi SS. The mitochondrial voltage-dependent anion channel 1 in tumor cells. Biochimica et biophysica acta. 2015; 1848:2547-2575.

81. Peng Z, Cheng Y, Tan BC, Kang L, Tian Z, Zhu Y, Zhang W, Liang Y, Hu X, Tan X, Guo J, Dong Z, Liang Y, Bao $\mathrm{L}$ and Wang J. Comprehensive analysis of RNA-Seq data reveals extensive RNA editing in a human transcriptome. Nature biotechnology. 2012; 30:253-260.

82. Kume H, Hino K, Galipon J and Ui-Tei K. A-to-I editing in the miRNA seed region regulates target mRNA selection and silencing efficiency. Nucleic acids research. 2014; 42:10050-10060.

83. Davey P, Rauth AM, Mason L and Addy L. Spontaneous phenotypic and karyotypic progression in the SV40 transfected cell line SVG during prolonged passage in vitro. Journal of neuro-oncology. 1990; 8:13-22.

84. Sonoda Y, Ozawa T, Hirose Y, Aldape KD, McMahon M, Berger MS and Pieper RO. Formation of intracranial tumors by genetically modified human astrocytes defines four pathways critical in the development of human anaplastic astrocytoma. Cancer Res. 2001; 61:4956-4960.

85. http://support.illumina.com/downloads/bcl2fastq conversion_software_184.html.

86. http://bio-bwa.sourceforge.net/.

87. Li H, Handsaker B, Wysoker A, Fennell T, Ruan J, Homer N, Marth G, Abecasis G, Durbin R and Genome Project Data Processing S. The Sequence Alignment/Map format and SAMtools. Bioinformatics. 2009; 25:2078-2079.

88. http://broadinstitute.github.io/picard/.

89. McKenna A, Hanna M, Banks E, Sivachenko A, Cibulskis K, Kernytsky A, Garimella K, Altshuler D, Gabriel S, Daly $\mathrm{M}$ and DePristo MA. The Genome Analysis Toolkit: a MapReduce framework for analyzing next-generation DNA sequencing data. Genome research. 2010; 20:1297-1303.

90. http://evs.gs.washington.edu/EVS. 
91. http://www.1000genomes.org/.

92. http://www.ncbi.nlm.nih.gov/SNP/.

93. Anders S, Pyl PT and Huber W. HTSeq-a Python framework to work with high-throughput sequencing data. Bioinformatics. 2015; 31:166-169.

94. Anders $\mathrm{S}$ and Huber W. Differential expression analysis for sequence count data. Genome biology. 2010; 11:R106.

95. Ramaswami G, Lin W, Piskol R, Tan MH, Davis C and Li JB. Accurate identification of human Alu and non-Alu RNA editing sites. Nature methods. 2012; 9:579-581.

96. Ramaswami G, Zhang R, Piskol R, Keegan LP, Deng P, O'Connell MA and Li JB. Identifying RNA editing sites using RNA sequencing data alone. Nature methods. 2013; 10:128-132.

97. Gao J, Aksoy BA, Dogrusoz U, Dresdner G, Gross B, Sumer SO, Sun Y, Jacobsen A, Sinha R, Larsson E, Cerami E, Sander C and Schultz N. Integrative analysis of complex cancer genomics and clinical profiles using the cBioPortal. Science signaling. 2013; 6:p11. 